\title{
LA DISSOLUTION DANS LA NATURE:ÉTUDE ÉCOLINUISTIQUE DE PRESSÉE DE VIVRE D"ANISE KOLTZ
}

\author{
Amgad El-Zarif Atta* \\ amgadelzarif71@gmail.com
}

Ce travail vise à scruter le langage poétique Anise Koltz en tant que poétesse qui se perd totalement dans le monde naturel. Il s'agit d'une enquête écolinguistique sur son dernier recueil Pressée de vivre (2018), qui préconise la philosophie écologique de la poétesse. Cet ouvrage poétique met en évidence l'engouement de la poétesse par la nature et révèle ses moyens écolinguistiques pour se sentir connectée au monde naturel. L'écopoétique s'oriente vers les problèmes écologiques du XXI siècle et traite le langage sous-tend des idéologies néfastes pour les écosystèmes contemporains. Il aborde aussi l'habileté de la poétesse à manipuler ses sens pour accéder au monde naturel et à utiliser des procédés langagiers pour façonner son art et capter son lecteur. Il met également en lumière son pouvoir descriptif et sa maîtrise de la création des paramètres linguistiques appropriés à son glissement continu d'un espace naturel à un autre. Bref, ce document tente d'examiner l'attitude d'Anise Koltz à l'égard de la nature, en plus de ses moyens de la reformuler, qui est au cœur d'une approche linguistique appelée écolinguistique.

Mots Clés: poésie, écolinguistique, monde naturel, pouvoir descriptif

* Professeur adjoint en linguistique Française -Chef du département de français et directeur du centre des langues et de la traduction Université d'Al-Arich 
"Où est la littérature qui donne expression à la nature? Il serait poète celui qui réussirait à mettre le vent et les fleuves à son service, à les faire parler pour lui" $\left({ }^{1}\right)$

\section{Introduction}

Le voyage de la poésie écologique d'Anise Koltz prévisualise tout le mouvement environnemental ; il s'agit sans aucun doute d'une présence pleine de vitalité et d'intensité. Dans ce cadre, la poétesse fait croître son écopoésie ; ce qui ne signifie pas la présence d'éléments de l'environnement et de la nature dans une pure présence physique, mais elle va au-delà pour voir le monde dans une perspective de connaissance environnementale.

Pressée de vivre (2018) (désormais PV), un recueil-album, est une enquête écologique qui favorise la joie sereine de la dissolution dans le monde naturel. Le recueil de Koltz met également en évidence des stratégies écolinguistiques qui font sentir la connexion à l'environnement naturel. Koltz y fournit la " brièveté, sobriété, crudité d'une parole méditante." (Martinez, 2006, 131) Elle le brosse "comme [un] enfant qui chante dans la cave plongée. " (Frank, 2001, 893) Son écopoétique serait ainsi "ce double élan de la (mise) en avant (du) monde naturel." (Schoentjes, 2015, 28)

Comme dans un texte écologique, Koltz pratique dans $P V$ une écriture hybride : "une combinaison entre histoire naturelle, autobiographie, philosophie et fiction ; [celle-ci] a évolué pour donner naissance à un genre à part entière." (Buell, 2002, 19)

L'écopoétique s'oriente restrictivement vers les problèmes écologiques du XXI siècle et traite "le langage [qui] sous-tend des idéologies néfastes pour nos écosystèmes." (Lechevrel, 2008,

( $\left.{ }^{1}\right)$ D.Thoreau, Walking, cité par Pierre Schoentjes, p. 23.

( LA DISSOLUTION DANS LA NATURE ...) Dr.Amgad El-Zarif Atta 1054 
26) L'écolinguistique va par conséquent "au-delà de la syntaxe, de la sémantique et de la pragmatique (en créant) des idées innovantes concernant la recherche empirique." (Fill et Mühlhäuster, 2001)

Koltz fonctionne ainsi le langage prémédité de $P V$ selon "les lois de la mimésis et de l'interaction" (Gomez, 2006, 128) avec le monde naturel. Ce mimesis "détermine la lecture du monde culturellement construite." (Okourou, 2018, 7) Koltz écrit " avec facilité, mais le problème, (lui) semble-t-il, c'est que (elle a) déjà dit tout ce que (elle) avait à dire." (Un entretien avec Grégory Cimatti le 11/06/2018)

Le fait de s'emparer de Koltz au monde naturel a pratiquement supposé plusieurs questions, puisque tout vol doit avoir des raisons qui se cristallisent dans un cadre écolinguistique : Un poète peut-il se perdre totalement dans l'environnement naturel et retrouver ensuite son individualité en tant que voix écologique distinguée? Et dans quelle mesure et par quels moyens? Pour apporter une réponse possible à ces questions, cette étude aborde Koltz en tant que poétesse qui vit dans et pour la nature.

Le choix de ce recueil a préconisé la philosophie écologique de la poétesse : réinvention, recréation ou réenchantement de la nature. Celui-ci "s'applique à l'aisthesis, c'est-à-dire à la capacité (ou l'incapacité) qu'a le texte littéraire de nous proposer un regard nouveau sur notre relation avec le monde naturel." (Pughe, 2005, 79)

Koltz prévisualise le mouvement environnemental pour figurer l'univers dont la plume favorise " une incitation à faire évoluer la pensée écologique, voire comme une expression de cette pensée." ( Blanc, alii, 2008/2, 15) Dans PV, la proximité 
directe entre le monde humain et celui naturel serait un "projet écosophique" (Pughe, 2005, 78) qui dépasse le clivage entre la culture de l'homme et celle de la nature, et décrit la propre façon d'habiter l'environnement naturel.

L'exploration de $P V$ passe sous l'axe théorique de Lawrence Buell $\left({ }^{1}\right)$, sans négliger l'apport important du pivot épistémologique de l'écopoétique de Pierre Schoentjes et les efforts de Michèle Monte de scruter la poésie contemporaine. Nous supposons que $P V$ forge le projet de la dissolution dans la nature à travers une quête à la recherche des éléments suivants :

\section{Structuration syntaxico-sémantique}

La structure des prédicats écolinguistiques (écogéographiques / écohistoriques) $\left(^{2}\right.$ ) révèle l'intégration dans l'environnement et dans le monde naturel. Le réseau de verbes écopoétiques glose la combinaison syntaxico-sémantique du langage poétique de Koltz: conjuguer les verbes en silence d'une force sensorielle naturelle.

\section{I.1. Les verbes écogéographiques}

Les verbes écogéographiques sont à l'origine d'un mélange et voire d'une fusion de la poétesse au monde naturel et à ses référents lexicaux : dessin du monde naturel privé, visualisualisation des représentations géométriques naturelles.

( $\left.{ }^{1}\right)$ Les critères essentiels d'un texte écologique sont les suivants: 1) l'environnement non humain est un acteur à part entière et non pas un cadre de l'expérience humaine; 2) les préoccupations environnementales se rangent légitimement à côté des préoccupations humaines; 3) la responsabilité environnementale fait partie de l'orientation éthique du texte; 4) le texte suggère lidée de la nature comme processus et non pas seulement comme cadre fixe de l'activité humaine. (Buell, et Suberchicot, 2002.)

$\left({ }^{2}\right)$ La dénomination de ces types de verbes de notre proposition. 
Dans la première scénographie ${ }^{1}$ du recueil à la page 9 , la poétesse s'est transformée en oiseau à l'intérieur d'un essaim migrateur. Koltz s'envole sur le bassin hydrographique du Nil, et "dévoile en elle la profondeur inconnue de sa véritable nature". Elle peut "se découvrir et se retrouver dans une forme de sérénité et de distance." (Forest, 1989 :97). Koltz déclare sa joie en disant:

\section{Majestueusement}

Le Nil traverse le paysage [...]

Je m'envole

Avec les migrateurs

Je m'intègre dans leur triangle ( $P V: 9)$

La poétesse s'efforce d'impliquer le lecteur dans la scène poétique par le biais des descriptions minutieusement détaillées. L'organisation de prédicats dans la scène a permis la flexibilité d'un mélange en douceur dans les essaims afin de décrire l'intégration totale avec les migratoires. Les trois verbes : traverse, m'envole et m'intègre ont régi la première scène naturelle. Ce choix raisonné "présente implicitement le problème environnemental de la pollution du Nil. L'adverbe de manière Majestueusement en tête de l'énoncé se réfère à la grandeur de ce fleuve, un roi couronné sur son trône, et réserve à la modification du prédicat traverse un "sémantisme actif et dynamique" (Van de Velde, 2009:15). Le prédicat traverse $\left(^{2}\right.$ ) souligne la capacité du Nil, l'agent de l'énoncé, à façonner le paysage à sa guise. La photographie aérienne provient de Koltz, un oiseau migrateur, pour ouvrir la vue de la cinématographie

( ${ }^{1}$ ) La scénographie joue le rôle d'un protagoniste de la représentation. C'est un choix de l'espace, son fonctionnement et son efficacité sont déterminants. Elle peut ainsi structurer la poétique construite autour du texte." Cf., (Pelechová, 2013/3 ( $\mathrm{N}^{\circ}$ 58).

$\left({ }^{2}\right)$ On note que la poétesse a utilisé le verbe traverser dans différents contextes au sein du recueil, qu'il soit géographique ou historique, voire figuratif pour incarner un pont magique pour l'errance dans divers espaces naturels. 
aérienne de la scène à l'intérieur de l'essaim. Le mélange a donné également à Koltz une vision surnaturelle que les êtres humains ne possèdent pas. Elle affirme sa nouvelle vision animalière en disant :

\section{Mes yeux creusant la distance}

\section{J'invente}

Un autre présent ( $P V: 46)$

Ce pouvoir extraordinaire lui a permis de posséder des « yeux » différents de ceux humains et de voir d'en haut; ce qui a donné à la poétesse le premier sens distinctif des oiseaux migrateurs : voir le monde avec les yeux des oiseaux du ciel, se confondre avec les migratoires et posséder le même sens afin de transmettre le même sentiment au lecteur.

Il est bien patent que les deux verbes pronominaux : "m'envole" et "m'intègre" ont un sens réflexif qui valorise la notion de la fusion. Le premier désigne la joie de la métamorphose avec de nouveaux membres "ailes", pour pouvoir partir avec les migrateurs. Le deuxième m'intègre, soigneusement choisi de Koltz, révèle son contact direct avec ces migrateurs, afin de multiplier le sens du monde naturel. Dans les deux cas, le pronom sujet "je" confirme l'implication de l'énonciatrice (=Koltz) dans la scène avec sa toute volonté dans cette fusion.

Le dessin de la première forme géométrique naturelle s'esquisse à l'aide du lexème triangle. Cette merveilleuse géométrie naturelle précise " le jeu réciproque entre le son et le sens" ; cela veut dire que certaines "présentations acoustiques capables d'être associées à des réorientations sémantiques." (Jakobson, 1963, 133) Les oiseaux migrateurs, caractérisés phonétiquement par les assonances constrictives de la consonne fricative [V], dans "traVerse, enVole, aVec", représentent un emblème du monde naturel. La forme orthographique de la lettre 
$V$ représente le sens de la géométrie du vol naturel et la propriété fricative du son qui incarne le sens du contact : les cris des oiseaux émis en vol les incitent à continuer le voyage. Cette image audio-visuelle des oiseaux migrateurs peut se comparer à celle de Kenneth dans son poème "Late August on the coast" ( Fin d'août sur la côte ») :

\begin{tabular}{|l|l|}
\hline $\begin{array}{l}\text { flies in regular chevron or } \\
\text { line formation flight note : aa- } \\
\text { honk }^{1}\end{array}$ & $\begin{array}{c}\text { vole en formation régulière, } \\
\end{array}$ \\
& V ou file \\
& \\
\hline
\end{tabular}

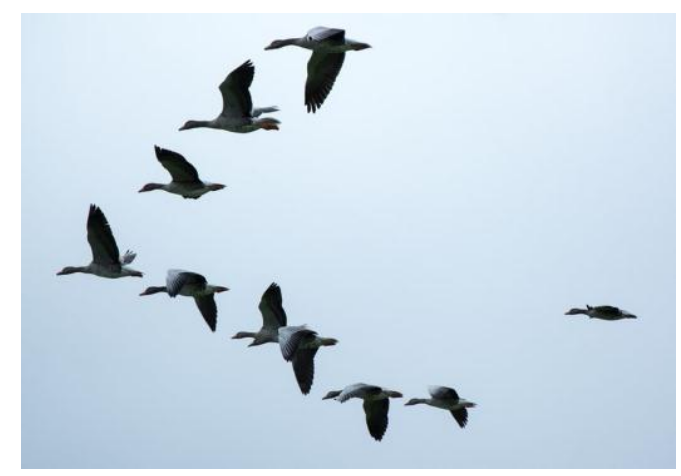

Figure naturelle ${ }^{1}$

Rompant la répartition de Benveniste (1974) des pronoms personnels, Koltz intègre le "je" de l'énonciateur avec le "leur" des migrateurs. Ce jeu syntaxique revêt, par les verbes géolinguistiques et leurs arguments, un merveilleux mélange avec la nature.

Ajoutons aussi que, les prépositions avec et dans placent également les oiseaux migratoires selon l'arrangement logique de l'intégration progressive. La préposition avec indique un manque d'adhérence totale à l'essaim et souligne qu'il y a encore une partie

1( (Kenneth White Atlantica, trad. Marie-Claude White, Paris, Grasset, 1986, p. 205.

( LA DISSOLUTION DANS LA NATURE ...) Dr.Amgad El-Zarif Atta 1059 
de la nature humaine chez la poétesse, tandis que la préposition dans rend plus sûr non seulement la connexion de la poétesse avec l'essaim, mais aussi sa réassurance dans cet essaim. Koltz traite délicatement le sens du toucher du lecteur et le fait synchroniser avec elle dans cette fusion avec les "voyageurs $d u$ ciel" (Zucca : 2015).

Koltz s'engage résolument dans un autre espace de son recueil-album pour se retrouver au hamac du soleil quand elle dit:

Je traîne dans le hamac

$\mathrm{Du}$ soleil balançant dans

Un vent apprivoisé (PV:12)

Koltz se remet à une autre scène de l'album afin de perdurer sa joie fantastique et dynamique au hamac du soleil. Afin de continuer sa joie fantastique et dynamique au hamac du soleil, une affusion calme avec les rayons du soleil crée un état de calme psychologique des vers à la scène céleste même si elle est séparée des oiseaux migrateurs.

Le soleil représente la force masculine capable de contrôler le corps de la poétesse, et le vent fournit l'émergence d'un mouvement confiant et calme qui génère son sentiment de confiance issu de son exposition aux cordes dorées du soleil. Le verbe traîner dépeint ce mouvement allongé et paresseux de la poétesse. Ensuite, l'adjectif postposé, apprivoisé, révèle la soumission de la nature à la poétesse en lui accordant un vent calme qui augmente sa sensation de confort et de sécurité. Voilà pourquoi Koltz exprime non seulement sa joie mais son extase résultant d'une " sensation d'une affinité biologique avec les éléments naturels" (Deschamps, 2018:157).

Remarquons également que Koltz, dessine régulièrement de nombreuses formes géométriques naturelles : la conception du Hamac prend une géométrie rectangulaire et sphérique. Les rayons du soleil de couleur dorée excitent à nouveau l'imagination 
visuelle du lecteur. Encore une fois, la préposition dans favorise la fusion de la poétesse avec la nature.

Le verbe traîner apparaît à nouveau pour nous amener à un paysage créatif et paradisiaque qui suggère l'éloignement total de la poétesse de la terre et son désir obstiné et urgent de s'accrocher au ciel:

Je traîne derrière moi la nuit

Avec son troupeau d'étoiles (PV: 59)

Des étoiles célestes nocturnes incarnent une configuration allégorique double : toucher les étoiles du ciel et naviguer dans un voyage nocturne dans le ciel engendrant tout un train d'images naturelles célestes nanties d'un mécanisme de mouvement comme une pellicule de cinéma. Koltz s'envole toujours dans le ciel, mais cette fois-ci pendant la nuit, en perdant à jamais son identité humaine déficiente et ayant une identité surhumaine sous de multiples formes. Cette flexibilité du mouvement extériorise le désir refoulé de s'échapper au monde humain vers le monde naturel.

L'énonciatrice se contente d'accompagner les étoiles et d'errer pour que le troupeau soit derrière elle. Koltz ne se lasse pas de se laisser conduire par cette force en flottant toute la nuit avec les étoiles. Nous constatons que l'incarnation a pris deux formes. La première est liée à la faune et représente la poétesse comme étant le chef d'un groupe d'animaux dessinés dans le ciel, image rendue par l'expression "chiens d'atterrage". La deuxième est inspirée d'ingénierie naturelle celle de «carré de Pégase» à l'automne ou celle de «triangle d'été» en été.

Éprise de la nature, Koltz trouve la clé de sa fusion en disant :

La neige me traverse

Je me sens plus légère

Que ses flocons. $\quad(P V: 88)$ 
Le lexème neige annonce explicitement l'hiver. La poétesse se transforme en une petite plante ou une feuille d'arbre tombée en automne. Si la neige est le sujet grammatical de l'énoncé, la sensation transmise à Koltz est directe par le contact direct entre la neige et son corps concrétisé par le verbe pronominal de sens réfléchi se sentir où le pronom complément $m e$ se réfère à la poétesse. Celui-ci porte le sentiment physique de la fusion avec la neige et reflète une impulsion irrésistible d'une intimité avec la nature.

La relation comparative de proportion plus... que, avec l'utilisation de l'adjectif légère, à sens susceptible d'être quantifié, débarrasse la poétesse, accablée de soucis et de fléaux psychologiques, de son poids physique. Cette transformation résout l'équation d'un corps humain lourd à travers le lexème significatif au pluriel, flocons. Le choix de ce lexème au pluriel inclut toutes les formes des cristaux de flocons s'effritant à jamais à cause du réchauffement climatique.

Ensuite, le verbe se réfugier, asile naturel, incarne la puissance marathonienne dans le transfert spatial au sein de la nature avec toute joie. Koltz annonce:

Je me réfugie parmi les pierres

Végétant dans leurs insomnies (PV:90)

Par conséquent, cette étape de mélange a donné l'occasion de tracer une ligne de mélange descendant avec la nature: du ciel à la terre jusqu'au fond de la terre. Ainsi, la poétesse se cache parmi les pierres et se transforme en réfugiée environnementale.

Ce nouvel amarrage d'un élément posé au sol fait ressentir la valeur de la matière minérale solide et dure. Une nouvelle vie de la poétesse trouvera son chemin, même parmi les pierres : ce lieu où elle se retire pour échapper au désagrément humain pour qu'elle puisse se mettre en sûreté. Le verbe se 
réfugier prend sa dimension politique : la haine l'Allemagne nazie, la torture et la mort de son mari.

L'image mentale naturelle est enveloppée du verbe végétant au participe présent qui rend apparente la poursuite du processus de croissance et de développement, c'est-à-dire l'enchevêtrement de la poétesse coincée avec des pierres. En outre, elle confère aux pierres des sensualités inquiétantes par le lexème insomnies au pluriel, nous rappelle le recueil de la poétesse "Un monde de pierres". (Koltz, 2015) Dans ce contexte, les prépositions parmi et dans crée l'organisation spatiale logique de la scène. La préposition parmi qui a le sens d' "au milieu de, au sein de" indique l'appartenance à un ensemble uniquement devant un nom pluriel ou un nom collectif assurant une transformation partielle, ce qui signifie que la plante n'a pas encore poussé. La préposition dans donne à la poétesse l'aisance de foisonner au cœur des pierres.

Soudain, Koltz se déplace le lecteur vers le désert au cœur de l'événement environnemental en disant:

Le désert me suit

$$
\text { Il est en moi }
$$

Mon autre vie m'abandonne

Je me remplis de sable (PV: 77)

Le désert, dépeint attentivement dans les pages du recueil aussi bien que d'autres vastes espaces écologiques tels que : ciel, forêt, océan, Nil ..., s'émerge avec des sentiments abstraits: la sérénité, la pureté pour montrer à nouveau le paradoxe sémantique (Riffaterre: 1971) entre le monde humain et le monde naturel à travers les deux verbes est en moi \# m'abandonne

Or, l'autre monde signifie toujours pour la poétesse sa vie humaine abandonnée. Le verbe m'abandonne accentue son énergie positive et ce grâce à l'effet de sa fusion dans le champ 
désertique. Sa vie humaine qui a été un fardeau est devenue plus légère au seuil du désert. Koltz annonce: "Mon autre vie m'abandonne au lieu de dire "j'abandonne mon autre vie". De même, " je me remplis de sable" au lieu de "le sable me remplit." L'effet de cette substitution, comme choix facultatif des arguments des énoncés, est donc d'introduire un lien d'intégration entre la poétesse et le désert, celui-ci est devenu une partie d'elle. Remplie de sable, elle devient une composante du désert.

L'énonciatrice stimule le sens tactile afin de s'identifier au sable. Ce choix explicite de couleurs jaune blanche, incarne la pureté de l'image du désert par opposition à la ville. La poétesse est alors dotée d'un nouvel avantage visuel surnaturel acquis du sable

La fusion de la poétesse la débarrasse de toute humanité afin de surprendre le lecteur avec le passage ci-dessus :

Je me promène en enfer

Comme aux cieux

Pour élargir

Mon après-monde

Il sentira soufre et encens (PV: 85)

Koltz déclare son renoncement de l'humanité et son acquisition d'une nouvelle âme et d'un nouveau corps errant en enfer. Je me promène en enfer fait référence à des relations sémantiques complexes: "je" souligne que la poétesse s'implique volontairement dans cet espace brûlant et infernal; me promène, s'adhérant à détendre l'âme dans un espace naturel agréable en dehors de toute limite. Or, Koltz étonne le lecteur en mentionnant qu'elle flâne en enfer. Il nous parait que l'image a été complétée dans la strophe ci-dessous qui a été coupée à deux reprises du recueil :

Derrière le ciel

Il y a un autre ciel (PV:9)

( LA DISSOLUTION DANS LA NATURE ...) Dr.Amgad El-Zarif Atta 


\section{[...] \\ Chacun de nous \\ Un déjà sa place \\ En enfer (PV: 19)}

L'écriture poétique environnementale intermittente met en abîme le lecteur dans un état d'excitation constante. La scène de l'enfer ajoute, d'ailleurs, des niveaux sensoriels et visuels à la scène à travers le soufre qui concrétise l'acuité visuelle d'une et l'encens qui dégage une merveilleuse atmosphère rituelle sur cet espace infernal.

Le voyage nocturne arrive bientôt à son terminal et les éléments que Koltz accompagne se retireront de la scène grâce à la brume matinale.

\section{Mon haleine se mélange \\ à la brume matinale (PV:76)}

Koltz insiste sur le fait que cet effort d'écoute monotone motive la conscience du lecteur à travers le verbe se mélange au sens pronominal. Ce choix lexical participe "d'une symphonie naturelle" (Poupon, 2018, 56) par le brassage entre l'âme de la poétesse et un élément de la nature.

En somme, les verbes géolinguistiques évoquent deux types d'espaces : le réel et le fictif dont la vocation souligne la description géographique du paysage, alors que les verbes écohistoriques vont évoquer, comme nous allons le voir, l'espace mythique (Barrucand, 2006, 33) dont la vocation est de donner des racines historiques à toute humanité. 


\section{I.2. Les verbes écohistoriques}

Dans PV, L'historicité $\left({ }^{1}\right)$ donne aux verbes écohistoriques l'occasion d'une nouvelle fusion naturelle. L'adhésion de l'espace mythique se manifeste dans le recueil dans deux cas: l'ancienne bibliothèque d'Alexandrie et la ville de Babel. Ces espaces historiques "se chargent de mémoire, d'imaginaire, de symboles [...] en dehors du savoir collectif." (Deschamps, 2018/13,158)

Tout compte fait, Koltz abandonne l'espace naturel pour se fondre dans l'espace historique. Le sème léternitél se déchiffre à maintes reprises: "je marche à travers les siècles" (PV:15) (bis, $P V: 54)$ "je marche vers une trompeuse éternité" (PV:37) "je marche pendant des éternités" (PV:131). Celui-ci s'associe à un mouvement rétrospectif : pharaonique et babylonienne, et retrace la poéticité de Koltz comme une page d'un livre imprégné de la bibliothèque d'Alexandrie, ou une partie d'une peinture murale dans les lois d'Hammourabi, un poème des écrivains du Livre des Morts, etc.

Koltz adopte son parcours historique en s'effaçant dans l'écho des noms propres:

Dernier jugement
devant Anubis
pesant mon cour
plus lourd qu'une plume. $(P V: 15)$

Le procès mythique du jugement de son âme devant Anubis, un affrontement judiciaire, porte des connotations sensorielles et picturales historiques : la couleur noire de sa peau résultant des effets de l'embaumement crée un paradoxe

$\left.{ }^{1}{ }^{1}\right)$ Rapportée à ce qui est spécifique, "l'historicité ne peut que renvoyer à la subjectivité, c'est-à-dire à un point de vue sur l'histoire qui ne soit pas dissocié des sujets humains" (Gérard Dessons 2005:264)

( LA DISSOLUTION DANS LA NATURE ...) Dr.Amgad El-Zarif Atta 
sémantique avec les couleurs vives (bleue, verte, blanche et jaune) du monde naturel dans le recueil.

Le nom propre Anubis, lié aux poèmes funéraires du livre des Morts, reflète l'obsession de la poétesse d'insérer sa poésie dans les recueils des morts des Pharaons. Le lexème "plume", fait allégorie à la plume de Maât ${ }^{1}$, représente le salut de Koltz au moment du jugement devant Anubis. Celui-ci accomplit la proximité acoustique avec le prénom de la poétesse Anise, notamment dans la première et la dernière syllabe. Supprimant les deux phonèmes [ub], les sons du prénom de la poétesse s'enracinent dans la mémoire du lecteur que Koltz adhère à l'âme de cet animal/dieu et son appartenance était l'écho mythique dans sa poésie.

Comment Koltz s'intègre-t-elle au monde historique? Un faisceau de verbes écohistoriques annonce, en cinq positions distinctes dans le recueil, les formes d'intégration dans le monde historique :

\section{Je descends de milliers d'ancêtres}

Qui végètent dans mon sang (PV: 17)

À ce niveau, la première scène historique, l'usage du verbe "descendre" (= venir "de" descendance), ayant une apparence figurative personnelle, précise la renonciation de la poétesse à ses origines européennes, mais surtout proclame l'enracinement de la vie de la poétesse dans des origines historiques très anciennes. La préposition "de" et le syntagme nominal " milliers d'ancêtres" contribuent à cette configuration d'une forte signification historique du verbe. Vient ensuite le verbe "végéter", utilisé dans un contexte géographique antérieur, annexé par un lexème adéquat sang. Celui-ci est le symbole de la parenté à cette de richesse culturelle pharaonique.

( $\left.{ }^{1}\right)$ Maât est, dans la mythologie égyptienne, la déesse de l'ordre, de l'équilibre du monde, de l'équité, de la paix, de la vérité et de la justice.

( LA DISSOLUTION DANS LA NATURE ...) Dr.Amgad El-Zarif Atta 
Dans la deuxième scène qui complète partiellement la première, Koltz annonce à deux reprises :

$$
\begin{gathered}
{[\ldots] \text { Vivre }} \\
\text { À travers la matière des ancêtres }(P V: 54)
\end{gathered}
$$

J'ai devancé ma vie

de mille ans (PV:64)

Le verbe vivre à l'infinitif retrace la transcendance déviée de la poétesse dans l'espace historique. Il explique également une partie du titre du recueil. Le localisateur à travers (= étendue transversale, largeur) ${ }^{1}$ suppose l'idée d'un (e) certain (e) assentiment, soumission, abdication ou d'une aide à franchir l'univers mythique. Cette combinaison avec le verbe vivre confirme que la poétesse franchit les barrières de l'espace et du temps non seulement avec ses pensées, mais aussi avec son corps quand elle cohabite avec ses ancêtres par l'émergence du syntagme "la matière de" en commutation phonétique avec le syntagme "la manière de": les paires minimales des consonnes [t] au lieu de [n]. Cette variante contextuelle historique de la matière se déchiffre sous ces différents sèmes :

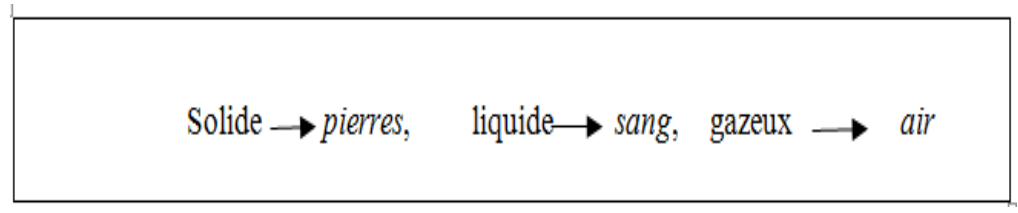

La réécriture de l'histoire passe par le verbe traverser. Or, elle est censée être élaborée à partir de son imagination plutôt que guidée par sa spéculation et son idéologie écopoétique. Koltz affirme que:

$$
\begin{gathered}
\text { Le sang qui me traverse } \\
\text { vient de loin }
\end{gathered}
$$

${ }^{1}$ ) Cf., Banque de dépannage linguistique, nuances sémantiques, FAQ, mars 2020.

( LA DISSOLUTION DANS LA NATURE ...) Dr.Amgad El-Zarif Atta 
Il charrie mes ancêtres (PV:67)

Le lexème sang revêt un goût prononcé d' "une recomposition littéraire qui alimente une topique du sang aristocratique sous-tendue par une orientation poétique délibérée." ( Giuliani, 2008, 227) La poétesse excelle au fonctionnement du verbe traverser dans deux contextes de fusion dans le monde naturel pour lui donner des dimensions sémantiques multiples. Cela signifie que le sang qui pénètre le corps de la poétesse vient "de loin" dans une référence explicite pharaonique. Sa persistance pour que le sang soit le premier fluide d'assimilation dans le monde historique, où elle s'incarne, et ce avec la présence de ce terme comme sujet dans le premier vers et à travers le pronom anaphorique "il " dans le deuxième vers.

L'autre verbe d'adhésion dans l'univers historique "charrier" renforce, d'autre part, le sang. Celui-ci transporte la poétesse dans un chariot: un moyen de transport antique primitif et proportionnel. Koltz abandonne toute sa timidité quand elle annonce:

\section{Je sculpte les paroles \\ Avec leurs échos \\ Depuis des millénaires \\ Le destin me fait naître \\ Et renaître $(P V: 82)$}

Koltz désire obsessionnellement éterniser sa poésie et la mélanger avec des symboles de l'écriture pharaonique. Le verbe sculpter rend de façon concrète la transformation de la poétesse en une statue de pierre à trois dimensions.

À suivre sa marche dans l'histoire, l'effet sonore de l'évocation ressort dans le syntagme leurs échos au pluriel. La poétesse ne renonce pas à fournir aux verbes des prépositions qui l'intègrent à l'histoire: les prépositions avec et depuis ajoutées au syntagme millénaires permettent au lecteur d'imaginer que 
Koltz a écrit ses poèmes avec la naissance de l'histoire pour devenir son propre esprit. Celles-ci évoquent également la référence spatiale et temporelle de la statue de la poétesse en tant que partie intégrante de l'histoire pharaonique.

Néanmoins, une telle volonté d'éminence historique souligne, de manière assez inattendue, son administration sur la conception même de l'idée d'adhésion charnelle. La poétesse est devenue un circuit intégré, et elle donne deux ou trois configurations exemples à cette fusion :

Mon image me couvre

[...]

Sur mon corps

Des signes d'anciennes civilisations (PV:25)

Un riche jeu de corps se manifeste entre la scène poétique et l'histoire confirmé et affiné par le verbe couvrir en tête de la strophe. Koltz met l'accent sur son état de soumission. Cette disposition élective que le lexème signes assume, est très puissant et illustre à quel point l'image poétique gravée précisément, de sorte que la poétesse a transformé son corps en une peinture murale.

C'est pour dire en somme que la première partie de l'intégration est centrée sur Babel en trois positions distinctes dans le recueil. Un langage poétique affirmatif assuré montre comment Koltz est obsédée par cette civilisation perdue quand elle dit :

\section{Babel}

Une fois de plus

S'écroule en moi

Avec fracas

$(P V: 34)$

L'intégrité se trouve remise en Babel. Le verbe s'écouler au sens passif suscite un choc pour le lecteur à travers les valeurs sémantiques et sonores qui s'adressent aux sens du lecteur (vue et ouïe). La préposition «en» et le pronom personnel «moi» 
renvoient à la contenance et à l'ampleur de la fresque (=le corps de la poétesse)

Le glissement du déictique temporel une fois de plus dans la scène démontre la nécessité de conjuguer le verbe à la voix passive afin de diriger la conscience et la réception du lecteur envers la valeur de l'image de Babel.

Or, ce processus ; le retour brutal à l'histoire, ne s'opère en toute sécurité, car il s'agit d'une violente fusion entre deux blocs. Il était nécessaire que la poétesse annexe la strophe par cette expression vocale forte avec fracas, qui effectue une perturbation intense et une horreur dans la scène. Cette collision conflictuelle aurait provoqué la tension et l'anxiété voulues pour capter les sentiments du lecteur.

Koltz avance la mise en abime innocente (Bauer, 2015:193) du jeu de l'écriture intermittente pour les feuilleter les ruines de Babel sous forme d'épigraphie poétique. On halète derrière la poétesse pour reformuler l'image entière car Koltz coupe la figure récurrente de Babel en deux parties: la première à la page 50 , et la seconde à la page 126 , presque à la fin du recueil.

Ce jeu est basé sur trois axes de dimensions énonciative, sémantique et lexicologique:

Premièrement: le jeu complexe des pronoms personnels dans:

$$
\begin{gathered}
\text { Je t'appelle Babylone } \\
\text { ressuscite } \\
{[\ldots]}
\end{gathered}
$$

de tes pierres pensives

de ton passé

$(P V: 50)$

La dimension énonciative s'inscrit d'abord dans la sphère poétique. Cette notion est bien confirmée par le pronom "te" et ses extensions "tes, ton, ta". L'énonciatrice se dirige directement vers 
Babel (= Babylone) qui exerce son autorité sur le destinateur indéterminé par le pronom nous.

Deuxièmement, la décomposition mythique de deux sèmes opposés /vie/ \# /mort/ se focalise aussi sur la manière de prendre en considération la "contradiction interne des entités du paradoxe sémantique" (Carel et Ducrot, 1999: 7) Récapitulée dans le tableau ci-dessous:

\begin{tabular}{|c|c|}
\hline Sème $/$ mort & Sème $/$ vie \\
\hline écroulés & ressuscite \\
\hline chute & Se redressent \\
\hline fouille & retrouve \\
\hline aveugles & pensives \\
\hline
\end{tabular}

Troisièmement, l'émergence d'une conclusion heureuse est à la prospection dans cet espace. Koltz annonce:

Déjà on trouve mes restes

Sous tes statues aveugles (PV:50)

$$
\text { [...] }
$$

Je fouille les ruines

Pour retrouver les vestiges

De même, quand Koltz évoque colonnes, statues, pierres, elle s'efforce de se retrouver parmi les vestiges de cet espace historique. Koltz creuse les vestiges de Babel pour mettre à découvert la grandeur de cette ville. Le syntagme pierres pensives, un usage métonymique de l'épithète, souligne que même si ses colonnes sont écoulées, et ses statues sont aveugles, elles peuvent ouvrir la voie vers cet espace légendaire majestueux.

En outre, la Bibliothèque d'Alexandrie illustre le deuxième témoin écohistorique. Stimulée par sa plume écopoétique, elle recompose un imaginaire poétique multiplié à 
cette époque. Le génie de cet espace historique excite l'inspiration écologique de la poétesse:

En feu la bibliothèque d'Alexandrie

L'alphabet se calcine

[...]

Mon nom tombe

En poussière

$(P V: 91)$

$$
\text { [...] }
$$

Décédée dans le feu d'Alexandrie

Je passe à travers les siècles.

(PV:110)

Les verbes écohistoriques se calcine, inonde, tombe incarnent une cartographie déterminée de cet horrible événement dans l'histoire de l'humanité. Le sème alphabet se réfère au sens collectif. Mais le verbe se calcine au sens passif de l'incendie) dépeint la destruction complète de cet édifice scientifique et académique. Concernant le verbe inonde, il démontre également le fait que la poétesse est détruite complètement comme une page dans l'un des livres de poésie de la bibliothèque.

Construits aussi sur un axe vertical, les lexèmes alphabet, mots, page, nom, qui s'attachent au même sème /historiographie/, encadrent les frontières brûlées de mots adossés au feu de l'incendie désigné par l'espace historique. La poétesse s'efface ainsi dans les plis des livres brûlés et se désigne par l'attribut décédée en tête du vers d'un énoncé elliptique. La fonction de localisation des prépositions en (= bis) et dans contrôle le mélange du corps de la poétesse sous les formes de la matière: feu et poussière.

Le sentiment d'enracinement historique dû aux matières primitives s'abrège dans les sèmes le feu, l'eau et la terre selon le triangle suivant: 


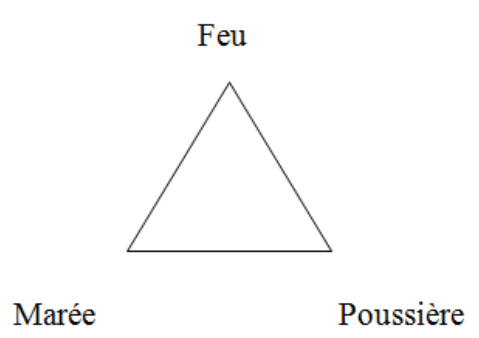

Ces matières nous amènent donc à contempler une essence glorieuse qui a ses racines dans le passé. Cette reformulation de la matière brute dans la poésie de Koltz crée une véracité établie avec la rigueur de la vision écohistorique. Elle amplifie avec joie dans sa poésie la conception de pureté de la matière considérée comme un signe distinctif de son originalité écopoétique.

\section{II.Procédés sémiostylistiques}

Si $P V$ est bondé de passages en langue figurative témoignant d'une résolution décisive de son projet poétique, tout ce que l'on retrouve souvent sous la plume des poètes écopoétiques, il y a pourtant des micro-éléments sémiostylistiques qui donnent une coloration naturelle à sa poésie.

Koltz redresse le rapport entre l'homme et l'environnement par le biais des procédés d'anthropomorphisme, de personnification et de zoomorphisme. Ceux-ci donne "une voix au monde aussi bien animal que végétal et [interroge] la place de l'homme dans les écosystèmes" (Buekens, 2019, 5)

Dans $P V$, les analogies "entre les registres animal, végétal et minéral rapprocher la nature du monde des lettres [effectuées] par le biais de la déshumanisation de l'homme ou de l'humanisation des animaux." (Deschamps 2018, 157) Koltz y accorde un statut privilégié à la question de la métaphore et à celle de la comparaison: une obsession écolinguistique stéréotypée développée systématiquement dans sa poésie. 


\section{IV.1 Voix de la nature : vers une métaphore écolinguistique}

La métaphore scrute la nature de l'image écopoétique "en tant que déviation sémantique du langage usuel et [mesure] le degré de poéticité de l'image." (Gomez, 2006, 132) Un réseau d'actes visuels dynamiques de mouvement flexible communique directement avec la nature, un rappel du film Avatar. Koltz s'étonne d'avoir des sens surnaturels, les voix de la nature.

Dans $P V$, la métaphore dite spatiale exalte "des abstractions [...] liées à la vue, à la localisation et aux déplacements" (Chanay et Rémi-Giraud, 2002, 79) en deux types : métaphores nominales, métaphores verbales

Koltz fait l'usage d'une métaphore au sens large : elle est une métaphore écologique globale. Celle-ci développe la diversité accordée à "sa force de persuasion et fécondité poétique." (Passeron, 2000, 13)

Koltz s'est effacée (s'est estompée) pour voir, entendre, sentir et toucher toutes ces chaînes de merveilleux ingrédients de la nature: la disparition suivie de l'absence de la première personne $J e$. Koltz a réinventé les mécanismes de l'imagerie esthétique pour reproduire la métaphore: une incarnation des sensations animées et vivantes.

La générosité illimitée de la nature a fourni à la poétesse son aisthesis (la recréation de sa nouvelle vision $d u$ monde naturel), elle s'interroge :

\section{Qui m'octroie \\ ailes et nageoires? (PV:103)}

Là, les ailes et les nageoires représentent des éléments incarnant les moyens naturels. À travers cette question dénonciatrice, la poétesse se demande quels sont les pouvoirs surnaturels qui ont provoqué la transformation de sa composition 
humaine en formes animales pour rédiger son script vert et tracer une voie alternative constituée d'un "imaginaire environnemental." (Buell, 1995, 35) La forme interrogative aiguise son extase symptomatique de se débarrasser de la nature humaine. Il semble ainsi que la contagion écologique de Koltz se soit déplacée dans le monde naturel à la suite de son adhésion à la nature. Les ailes et les nageoires cultivent, en retour à la nature, le vif désir et le bonheur intense d'un libre mouvement.

\section{IV.I.1 La métaphore maritime}

De manière essentielle, la métaphore de l'espace maritime exploite brièvement les décalages pour doux vs salé, petit vs grand, et calme vs agité. (De Chanay, Rémi-Giraud, 2000, 80) La surface du Nil, l'agent de la métaphore, s'associe aux eaux calmes, espace étroit (par rapport à l'océan) pour incarner au début du recueil. Koltz annonce:

L'éternité se fait porter par ses flots

Avec son pinceau d'ombre

Le soleil peint

Sur chaque surface

Des fragments

D'un monde englouti (PV: 10)

Pour se référer au sacrifice et à l'abnégation de ce grand fleuve, la métaphore se porte sur se faire porter, référent à l'idée du travail forcé. Le lexème ombre d'une couleur noire implicite, renforce la conception de la fertilité d'alluvion et la structure conceptuelle iconique du Nil. Ajoutons qu'une intensité vocalique se nait de la répétition de voyelles très proches [e] fermée, $[E]$ ouverte, [ə] muette dans éternité, se fait, ses, avec, le aussi que la voyelle [o] orale dans porter, flots, pinceau, et [Õ̃] nasale dans ombre, son, monde. Ces allitérations sont un medium sonore favorable pour amener le Nil avec son pinceau d'ombre à la 
créativité naturelle. Un rapport de conformité, de symétrie métaphorique explore le décalage et l'ancienneté du Nil dans le syntagme monde englouti.

Cette perception est cultivée par le verbe peint rappelant à la mosaïque de Palestrina. Cette action apparaît à partir de trois syntagmes géographiques et historiques: Chaque surface, fragments, monde englouti.

Or, l'océan incarne l'aspect intransigeant de décalage de la métaphore maritime: une amplitude et une salinité. Ces éléments océaniques sont planifiés dans deux scènes distinctes. Dans la première scène, Koltz dit:

L'océan se jette

Contre les falaises

Sa cervelle explose

En mille éclats. (PV: 13)

Deux verbes d'agitation se jette, explose rendent la sensation forte par l'effet d'un heurt matériel. Le verbe se jeter qui illustre le mécanisme du mouvement de collision sans retenue et sans arrêt contre la très forte formation rocheuse des falaises. Quant au syntagme en mille éclats, il ajoute la dimension quantitative répétitive de la métaphore océanique d'une manière distinctive.

De plus, la préposition contre indique un sentiment d'hostilité et une concurrence intense entre les parties de la métaphore. Les blocs sémantiques contradictoires sont ainsi transformés en masses sonores rivales grâce à la distinction vocale et nette entre les consonnes $[\mathrm{s}]$ et $[\mathrm{z}]$. Ce niveau de sonorité de la métaphore, d'harmonie imitative, s'entend intensivement du bruit fort de la folie de l'océan. Les sonorités sifflantes dans les variantes spécifiques océan, se, falaises, sa, cervelle, explose reproduisent les voix de "Monsieur" l'océan, 
chef d'orchestre, en rage; cet usage organisé de l'allitération bourdonne le délire terrifiant de l'océan et causant du mal à la cervelle.

L'incarnation métonymique de l'océan apparaît en sa cervelle afin de déchiffrer le caractère basique de la métaphore. (De Chanay, Rémi-Giraud, 2000, 80) Le verbe explose spécifie une résistance permanente vaine des roches des falaises, sans arrêt par l'érosion, disposées horizontalement pour juste créer une entrée encadrée de piliers de roches dures qui limitent le déplacement du mouvement vertical de l'océan.

Aussi, le rapprochement métaphorique océanique revêtil trois types de fonctionnements : substantielle, structurelle et acoustique. Le fonctionnement substantiel, sous forme d'une liste de syntagmes: (mer, images d'éternité), (chants sauvages, de l'océan), (les ombres du soir, visages effacés), crée le caractère d'analogie entre les entités de la métaphore. Le fonctionnement structurel s'organise dans traversent, coupant, étale, écoutent. La métaphore reste basée sur le mouvement de l'imagerie synthétique pour peindre une saillance visuelle romantique rarement vue dans $P V$.

$\mathrm{Au}$ niveau acoustique, les voix des éléments maritimes se sont engagées comme une cithare reflétant l'état calme de la mer/océan. L'allitération porte cette harmonie consonantique à travers deux consonnes: la consonne liquide [1] dans Le, ciel, l'air, leurs, ailes, déployées, La, les, laquelle fait entendre phonétiquement le mouvement des vagues calmes en contact avec la plage ainsi que la consonne [k] dans Quelques (bis), coupant, écoutent, fait découvrir un fond sonore fort qui permet au lecteur d'apprécier les voix des mouettes au-dessus de l'océan. Ces notes acoustiques de l'orchestre naturel sont magnifiquement tissées dans le syntagme océanique chants sauvages afin que les 
fonctionnements substantiel, structurel et acoustique prennent leur prééminence axiologique. (De Chanay, Rémi-Giraud, 2002: 84)

\section{IV.1.2 La métaphore désertique}

Le fait de "se réconcilier avec le désert comme métaphore d'errance et de quête de soi, c'est se réconcilier avec l'essence amphibologique de l'être humain", Atmane Bissani: 2010). La métaphore du désert repose sur "sa réalité conceptuelle signifiée". (De Chanay, Rémi-Giraud, 2002, 58) Elle déclenche une partie de la philosophie de Koltz dont le langage est sauvage, pur et intact. Koltz navigue en télescopage au fond du désert en s'appuyant sur une expérience intrinsèque de ses composants matériels: soleil, vent, caravanes, chameaux, vacarmes, tempête, sècheresse, sable et spirituels: calme, solitude afin de partager les sentiments avec lui. Dans une image proche du surréalisme, la poétesse a ouvert la voie à l'errance au fond du désert en disant:

Dans les yeux de mes enfants

Je voyais partir des caravanes

La métaphore passe d'un espace exposé aux yeux des enfants et réalise un alliage d'analogie du sème commun /puretél entre enfance et désert. Ce départ imaginaire mène au concept de virginité, d'innocence, de pureté et de sérénité et ce en liant ce désert à l'enfance.

Pourtant, dès que Koltz vit l'expérience du désert, elle se heurte à une expérience existentielle. L'équation métaphorique change complètement, pour que nous nous retrouvions impliqués avec elle dans un espace hors langue quand Koltz montre :

Dans ce silence

Végète le vacarme

( LA DISSOLUTION DANS LA NATURE ...) Dr.Amgad El-Zarif Atta 1079 
Dans la tempête

Le calme

Dans le terrent

La sècheresse (PV:107)

La métaphore siège directement dans la verticalité de la caravane comme si cette dernière devenait le véhicule du parcours spatial de la découverte des secrets du désert ; métaphore illustrée dans la figure ci-dessous :

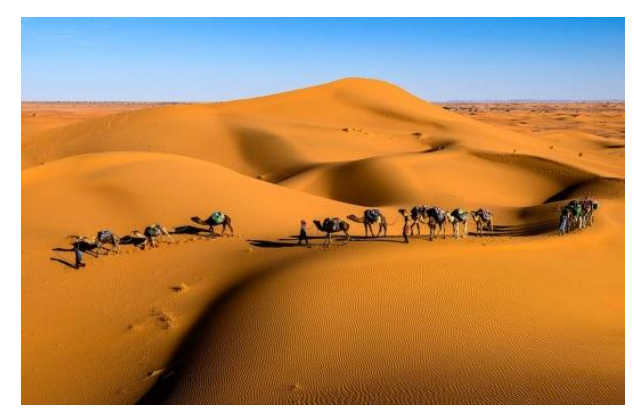

Figure naturelle VIII

Le sème /mutité/ du désert domine la strophe citée làhaut qui se trouve renfermée dans le silence=le calme. Pourtant, le paradoxe sémantique s'émerge clairement dans chaque pas de la caravane et suggère que le désert offre "une recette (qui) ne lésine pas sur les ingrédients contradictoires" et que cet espace arrange l'axiologie du message poétique "tant dans l'image que dans le texte : c'est là que vont s'entrelacer l'analogie et l'homologie" (De Chanay, Rémi-Giraud, 87)

Ce climat révèle la déviation météorologique capricieuse du désert. Koltz a lié les caractéristiques du désert aux changements climatiques grâce à une équation linguistique mathématique. Comme dans l'analogie paradoxale, quatre lexèmes verticaux (vacarme \# tempête), (torrent \# sècheresse) 
annoncent l'isotopie spatiale patente de la position sur l'axe de la verticalité de la métaphore. Le verbe végéter se dresse au sommet du passage comme une bosse de chameau qui stocke non seulement de la nourriture mais aussi des souvenirs et des expériences $\mathrm{du}$ désert. Nous pouvons ainsi réorganiser les relations métaphoriques du climat du désert selon le tableau cidessous:

\begin{tabular}{|c|c|}
\hline Etat permanant du désert & Changement climatique \\
\hline Silence & Vacarme \\
\hline calme & Tempête \\
\hline sècheresse & le torrent \\
\hline
\end{tabular}

Le caractère symptomatique et prévisionnel de la métaphore fait qu'elle est relativement liée aux lexèmes volcan, tempête, torrent, lesquels porte la cruauté et la terreur dans leur apparence. Cependant, ces phénomènes naturels éphémères représentent un stimulus sensoriel et un indice naturel de la Pluie probable. Koltz est un géologue habile dans les énigmes du désert dont elle utilise les éléments intrinsèques simples et contradictoires dans une représentation dynamique de l'image du désert.

Dans ce vaste vide, la métaphore spatiale du désert n'est pas apprivoisée dont la scansion se réfère à un réseau quantitatif ou qualitatif : le vacarme est aléatoire, d'un écho naturel subit, la tempête s'oriente de haut en bas, le volcan est, au contraire, de bas en haut. Ceux-ci secouent le vide du désert. La vision dynamique de la métaphore produit un regard abyssal, et prédit un changement de l'état de sommeil dans le désert.

La valorisation de la métaphore du soleil est étalée sur deux positions du recueil : 


\section{Le soleil s'appuie}

Sur moi

Le ciel fatigué

Ne sait où se reposer (PV:103)

$$
[\ldots]
$$

La main lourde du soleil

Se repose sur ta nuque

La chaleur se déchaine

Le temps

Suit les chameaux

Qui traversent lentement

Le désert

(PV:129)

Pour le choix de l'horizontalité, le verbe s'appuie localise la métaphore de la disposition horizontale du soleil. Toutefois, ce verbe provoque la métamorphose dans la puissance royale du soleil puisqu'il se tient fortement à l'énonciatrice. Ce rapprochement corporel entre le soleil, signe de la force mâle, et la poétesse assoiffée de tendresse, donne à la métaphore une dimension sensuelle et émotionnelle. Mais ce Roi soleil cherche toujours sa dominance à travers la température chaude d'où le verbe se déchaine intensifie la mise en colère, la maîtrise de la chaleur solaire sur le désert.

Les termes soleil, désert et ciel organisent le tactile du cadre spatial ouvert du désert. Alors que le déictique "le temps" correspond à l'arrangement naturel de la journée où le soleil règne sur le cadre spatio-temporel du désert. Et les animaux, qui s'y sont réfugiés, sont à la merci de leur énergie, sauf "les chameaux". La poétesse a dépeint les chameaux comme le navire du désert, lesquels résistent au roi sans indifférence. L'homologie du pur espace différentiel du désert parait subjectif si le verbe traverser 
figure la proportionnalité de la vacuité désertique de la métaphore. L'ajout qualitatif, à travers l'adverbe de manière "lentement", caractérise le mode synchronique du mouvement de la caravane mentionnée au début du recueil afin que l'image, mise en abime, soit claire et complète au terminus du trajet au désert.

Il semble nettement que le désert prête gratuitement au vent la délégation législative discrète sur son espace :

\section{Le vent écrit ses lois \\ dans le désert \\ s'associant à la solitude}

Du sable.

(PV: 117)

Le verbe écrire repère une double compatibilité avec ses arguments: son sujet le vent, et son complément de lieu dans le désert, tous les deux concrets. Alors, le vent, un législateur, promulgue les lois du désert qui régule le mouvement des créatures et des plantes. La métaphore verbale, transférée de la signification de écrire à la reconnaissance des processus morphologiques, conceptualise l'enrichissement analogique de l'image du vent. Pour cela, la représentation métaphorique unit une relation dynamique du verbe de mouvement naturel organisé. Le vent entreprend également de libérer l'imagination environnementale afin de privilégier le caractère obligatoire du vent aux détails du désert où cette description du mécanisme du vent s'intitule les lois de la dynamique des fluides.

Un travail effectué sans relâche et dans une activité excessive, la construction verbale au participe présent S'associant $d u$ sable visualise la plume par laquelle le vent écrit ses lois. Pour que le vent soit une nouvelle voix dans la symphonie de la nature harmonieuse, la consonne [s] reproduit non seulement acoustiquement le mouvement et l'activité du vent au cours de sa 
mobilité quotidienne organisée, mais est également considérée comme une force lyrique stimulant l'énergie du vent et le fait sortir de sa stabilité.

Quant au trait temporel relatif au mouvement du vent la poétesse précise une temporalité longue d'une expressivité maximale en citant:

\author{
Le jour se désagrège lentement \\ un vent abandonné \\ chasse tout ce qui bouge \\ par terre \\ dans les arbres (PV: 128)
}

La métaphore reste verbale avec se désagrège se référant à la longueur de la journée dans le désert; le monde désertique se déplace chroniquement en contact avec terre et arbres. Les propriétés du vent créent une résonance appréhendée aux verbes chasse et bouge de manière significative: Koltz incarne la forte activité des vents dans le désert d'une manière vivante et écologique. Une expérience vécue régulièrement renferme une exaltation d'un fort sentiment de puissance au point que "la métaphore verbale a le pouvoir de se propager aux actants. " (Chanay et Rémi-Giraud, 99) Cette puissante unité organique décrit la progression monotone avec l'adverbe de manière lentement qui fait entendre la présence du vent de manière pleine et circonstanciée dans le désert.

\title{
IV.1.3. Métaphore céleste
}

Initialement, la métaphore verbale céleste, illustrée par marchant, revêt un mouvement errant sans but. Koltz dit:

Marchant sans but 
d'un horizon à l'autre

les lettres de l'alphabet

nous poursuivent

Elles échappent

à la loi de la pesanteur. (PV:89)

Cette structure spatiale étonnante d'un horizon à l'autre dépendait du contraste cinétique entre l'espace terrestre vertical et celui horizontal céleste. Cependant, la poétesse entame une entrée libre dans cet espace ultime. Elle s'évade avec ses outils de création poétique. Cette métaphore contient une synecdoque convoquée référentiellement par les lettres de l'alphabet pour souligner l'un des traits d'une relation partie/tout de la poésie de Koltz et donc ce mélange allégorique avec les éléments célestes.

À l'appui de cette analyse, le verbe marcher qui est une affaire terrestre, se lie toujours à la loi de la pesanteur. Il est "en accord avec le contexte, en faisant appel à des "doubles cohérents" tels qu'évoluer, progresser, tendre, qui dénotent un processus de nature temporelle" (Chanay et Rémi-Giraud, 97) : le sens métaphorique se dirige vers un viol et une agression temporelle volontiers concernant le traitement de la métaphore.

La conception de cohérence temporelle représente l'organisation chronologique de la métaphore à travers cette série de verbes de mouvement marchant, poursuivent, échappent. Ensuite, la manière accélérée du déplacement dans le ciel sans jambes ni pieds se heurte très exactement au contenu sémantique du verbe marcher. L'action illimitée de navigation implique des mouvements illimités qui assurent le déséquilibre du corps humain et qui se reproduit à intervalles irréguliers au cours d'une progression continue dans le ciel. La localisation du mouvement céleste souligne qu'il s'agit du mode d'athétose fantastique de 
Koltz dans un décollement avec le sol dans son déséquilibre psychique.

Tous ces traits caractérisent l'effet analogique qu'apporterait cet enchaînement temporel des verbes loin de l'attraction (= le référentiel terrestre) qui exerce la loi de la pesanteur sur le corps de la poétesse. La plantation du syntagme la loi de la pesanteur au bout de la strophe (de la page) annule la loi universelle de la gravitation ou loi de l'attraction universelle, d'Isaac Newton, pour donner carte blanche à Koltz pour une liberté d'entremêlement avec le mouvement des corps célestes.

D'ailleurs, le mouvement spatial s'accélère lorsqu'un nouveau composant des éléments célestes entre dans la relation métaphorique imbriquée. Koltz affirme:

$$
\begin{gathered}
\text { Des nuages circulent } \\
\text { Sur ton plateau de solitude } \\
\text { qui soutient } \\
\text { lumière du jour } \\
\text { et de nuit } \\
\text { nourrissant illusions } \\
\text { et désillusions (PV:86) }
\end{gathered}
$$

Le processus métaphorique s'accentue comme une forme optique dans laquelle le verbe circuler se met au service du lecteur. Koltz reformule une certaine hiérarchisation verbale des traits propriétaires de la métaphore dans soutenir, nourrir: le premier consolide le rapport du toucher céleste entre la poétesse et les nuages alors que le deuxième favorise l'absorption ou l'allaitement au sein de la nature/mère. L'analogie s'empare des valeurs sensuelles. Le mouvement diurne du ciel s'accentue avec le verbe circuler. Ainsi, les nuages incarnent une sorte d'illusion 
visuelle factice concrétisant, dans deux cas, le paradoxe sémantique binaire : temporel entre jour \# nuit et psychologique entre illusions \# désillusions.

Ce chaosmos de la métaphore spatiale met surtout en jeu deux autres formes d'illusion macropsique de la propriété de l'espace céleste : au niveau chromatique, à travers les couleurs des nuages blancs et bleus, et au niveau cinétique par l'accélération du mouvement organisé des nuages. La métaphore crée une suspension du regard au ciel, pour que le syntagme nominal plateau de solitude basé sur un oxymore (nom + nom) de paradoxe analogique dû à une alliance de deux mots plateau + solitude. Ce paradoxe se trouve rapproché et combiné de manière que, "tout en semblant se combattre et s'exclure réciproquement," il frappe "l'intelligence par le plus étonnant accord, et produit le sens le plus vrai, comme le plus profond et le plus énergique." (Monte, 2007:137)

Dans le cadre de cette flânerie céleste allégorique, imaginée d'en haut, Koltz se retrouve sous l'influence d'un nouvel élément de la métaphore du ciel nocturne, une étoile errante:

Une étoile errante

m'a prit

sous son signe. $(P V: 16)$

La conceptuelle nocturne contraste avec la scène diurne des nuages par la présence d'un sujet céleste, l'étoile devenant un acteur contrôlant la poétesse emmenée dans un monde magique. La métaphore céleste détermine un espace nocturne de déplacement par rapport à la finalité de l'image. Un mouvement métaphysique du verbe prendre, en gros plan, se traduit, au plan syntaxique, par la fréquence des compléments d'accompagnement sous son signe. Une illustration spatiale montre l'étoile mère qui 
intègre la poétesse dans son "orbite"; cette étoile accompagne la poétesse pillée dans une excursion d'astronomie. Koltz "établit une relation avec l'espace à travers ses pensées, ses émotions et ses expériences sensorielles." (Buekens, 2019, 4)

Cette dernière et ultime réorganisation de l'espace céleste en forme de spirale nous amène au fond du ciel en hibernation provisoire ; rêveuse, Koltz en avoue :

En rêve, je retrouve parfois

le nébuleux passage des morts

qui monte vers le ciel

lieu de rencontre des justes se parvenant avec leurs aurédes (PV:81)

En effet, dans cet espace céleste ou stellaire, Koltz change de silhouette, elle prend une forme spirale dans une allégorie à son recueil Galaxies antérieures où "on en découvrira un nouvel aspect dans cet ensemble de poèmes brefs et denses." (Jossua, 2014/1, Tome, 98) Cette émergence se précise de l'horizontalité dans deux constructions verbales: monte vers, se parvenant avec. Aussi, la notion du temps est-elle forcément abolie par l'imagination de "en rêve". Le concept du rêve exécute ainsi une rupture des barrières du pouvoir temporel humain. Il s'agit donc de supposer qu'elle a fortement détruit les frontières du temps et de l'espace associées à la loi de la pesanteur. Le lexème nébuleux est un terme polysémique d'une ambiguïté lexicale dont le contexte poétique peut bien déchiffrer le sens adhérant. Comme nom, il précise un espace astronomie, un objet céleste composé ; mais comme adjectif, il signifie un obscurci par les nuages, qui manque de clarté. Tous les deux dégagent une impression d'un objet du ciel d'aspect diffus dans le rêve. 
Koltz perçoit à travers les yeux les morts dont l'invocation est conditionnée par le rêve. Cet espace céleste "se présente [...] submergé par une atmosphère de tension [pour indiquer] la nécessité de respirer contre le monde humain." (Martínez, 2006,136) Le fonctionnement métaphorique céleste s'étend enfin aux termes justes et aurédes dont le premier propage une chaleur et une luminosité à la scène du ciel nocturne, et le deuxième éclaire la scène à travers des sens radiologiques et religieux et la rend majestueuse.

\section{IV.2 La comparaison : énonciation écologique}

Disons que, dans PV, la comparaison est l'énonciation poétique d'un sème thématique commun, vraisemblablement écologique, à deux lexèmes différents. L'adverbe de comparaison "comme" et la locution "tel que" sont à titre de représentant de l'élément comparatif de la nature. Koltz respecte normalement la forme canonique de la comparaison ": A est B comme $C$ où $B$ est le prédicat ou sème commun aux deux lexèmes dont l'un, $A$, est le " comparé » et l'autre, C, le « comparant»." (Cohen, 1968, 44) Cette comparaison naturelle avec son fameux outil comme a créé intensivement un stéréotype naturel d'une forte occurrence dans $P V$. Koltz s'est remuée dans ses poèmes comme une comparatiste en cadence avec de longues ondulations écologiques. Ce remuement tire la poétesse dans trois orientations du déplacement réorganisées en trois points successifs:

Vitalité de la comparaison: la nature, un acteur rebelle

L'éthos de la poétesse en face de la nature

Évocation de l'Autre: la nature, un médiateur spirituel 
Son goût écopoétique excessif transfert au comparé: nature, Ethos, René d'une valeur sensorielle au comparant naturel: minéral, végétal, maritime. Les qualités naturelles du comparant sont dirigées vers le lecteur: la hiérarchie des images propres au contenu des termes comparés semble nécessaire à la perception de cette assimilation avec les écosystèmes.

Dans les trois cas mentionnés là-haut, le rapprochement, dépendant essentiellement de "l'impertinence", est adopté par Cohen et désigne "l'incapacité d'un terme à remplir sa fonction prédicative." (1968, 47) Dans ce cas-là, le prédicat (= le verbe de l'énoncé poétique) qui peut être "un fait, un évènement, une action, une activité, un état, un processus, une propriété, une relation, une quantité" (Mel'čuk et Polguère, 2008,100), joue un rôle majeur dans l'interprétation de la comparaison. Il est exclu par le comparant, le comparé ou les deux.

\section{IV.2.1 Vitalité de la comparaison : effet évocateur de la nature rebelle}

Dans le premier remuement, Koltz guette attentivement la nature avec frénésie, lorsqu'elle dessine le comparé (= thème) en face d'un comparant (= phare) (naturel, animal, humain ou concept): nous notons que trois prédicats soutiennent l'une des formes évocatrices de la comparaison dans trois positions du recueil :

- Les nuages passent comme des obstacles

à peine visible (PV: 115)

- qu'il (le vent) chasse (le sable) devant lui

comme une meute de chiens (PV:117)

- tout change comme des nuages qui nous

surplombent et qui s'en vont (PV:124) 
Vivre dans une vaste réserve naturelle fait apparaitre l'effet d'exaltation de la nature dans un état comparatif et proche avec différents éléments matériels qui représentent la formation physique des nuages dans les obstacles ou la représentation figurative des nuages en meutes de chiens. La comparaison place des nuages dans le cas du comparant mais l'avance progressivement vers «le comparé » pour que l'énonciatrice démontre que la nature seule puisse créer sa propre humeur sans aucune intervention humaine. Les deux verbes "change", "surplombent" figurent les processus physiques de formation des nuages, alors que les deux verbes "passent", et "s'en va" réorientent alternativement l'itinéraire de leurs processus dynamiques.

Contrairement à Baudelaire, partagée entre l'effacement de soi et la grande excitation de l'esprit, Koltz émancipe le vent comme un effet sonore de cette scène diurne et un accélérateur du mouvement terrestre de la planète associée au mouvement céleste des nuages. Celui-ci est similaire aux sons des meutes de chiens en voyage de chasse. La poétesse compare le son du vent fort aux sons des chiens sauvages dans un phonème naturel complet de comparaison: l'analogie acoustique d'un élément naturel avec un autre élément naturel manifestée dans l'allitération de la consonne sonore [v] dans visible, vent, devant, vont.

Koltz s'active avec le mouvement diurne caché derrière le rayonnement du soleil. Son génie écopoétique réapparaît à travers la création et la sauvegarde d'une image d'analogie extrêmement convaincante lorsqu'elle dit:

\section{Lorsque la lumière \\ dévale des hauteurs \\ et glisse sur ma peau \\ Le soir s'évanouit \\ devant ma porte}

( LA DISSOLUTION DANS LA NATURE ...) Dr.Amgad El-Zarif Atta 1091 
La lumière du soleil descendant des hauteurs sur la peau de la poétesse représente la stimulation sensuelle et tactile superposée et adjacente à son corps. Le déplacement naturel du mouvement de la nuit et du jour représenté à travers une série de verbes cinétiques d'un mouvement escarpé, imprévu, foudroyant: dévale, glisse, s'évanouit. Avec un lever timide du soleil, la nuit est réinsérée pour qu'elle disparaisse complètement, et que l'image analogique termine la nuit par la mort symbolique; un cycle fatal et paradoxal. Le soir se fane devant la porte silencieuse de la poétesse comme la mort imprévue. La structure picturale comparative de l'image naturelle se complique dans la scène poétique de sorte que nous puissions en tirer trois figures parallèles: allégorie, métaphore et comparaison comme suit:

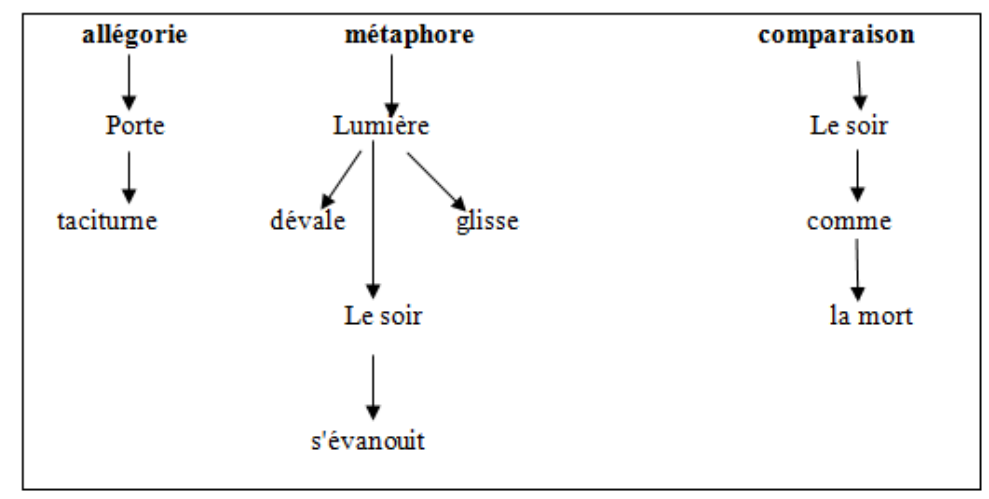

Cette vue extrêmement spectaculaire du soleil en cas de vieillesse fait entrer la poétesse par la porte du soleil dans le monde nocturne afin de solliciter de nouveaux joueurs en cas d'activité différente et renouvelée. La structure de l'image koltzienne s'embrouille par la complexité allégorique. Cet acte intentionnel vise à deux conclusions: la création d'un état de forte 
personnalisation du soleil; et l'occurrence d'un état de contact chaleureux entre le soleil et la poétesse. Koltz l'affirme en disant:

Dans mes poèmes

L'atmosphère retentit

Comme un tambour

Que personne n'entend

mais qui bat comme une métaphore

du silence (PV:126)

$\mathrm{Au}$ bout du recueil, Koltz accueille une comparaison extrêmement impressionnante et complexe, combinée à la page 126, presqu'à la fin du recueil. Elle crée une puissante contrainte de convergence avec le monde naturel représenté par le lexème atmosphère qui récapitule les éléments d'images intermittentes et dispersées de la nature, tels que le soleil, le vent, l'air, la vapeur, la lune etc. La poétesse abrège les éléments écopoétiques avec un seul terme au singulier qui inclut tous les éléments du monde naturel dans $\mathrm{PV}$.

Or, Koltz n'était pas satisfaite de cette proximité avec le monde naturel, et elle a ajouté une nouvelle dimension à la comparaison en liant sa parole à la musique de Gabriel Urgel Reyes (2018) dans son album musical une métaphore du silence. Si le lexème "tambour et les verbes sonores retentit + bat appartiennent à l'univers de la musique électronique de Urgel Reyes, les mêmes termes jouent de la musique chez Koltz dans les consonnes de battement [p] et [b] dans : poèmes, tambour, personne, bat à l'alternance rythmique interne. La comparaison relie le renouvellement de la poésie postmoderne à la redéfinition de la musique classique. Il ne s'agit pas de perdre de vue de la sélection rigoureuse de la magnifique structure "une métaphore 
du silence $^{\prime \prime}$ titre de l'album de musique sorti la même année que le recueil Pressée de vivre 2018.

Ajoutons que l'impertinence sémantique du terme silence crée un "hypallage" qui vise à " provoquer la surprise, le frisson de la tension paradoxale" (Molino, Gardes-Tamine, 1982, 204/205). Ce choix sémantique souligne l'étendue de la passion de Koltz, qui se dissout à l'aide des effets audiovisuels des éléments naturels. La corrélation comparative entre les composants naturels et les verbes de cette relation se résume ainsi selon le modèle de Cohen (1968) :

\begin{tabular}{|l|l|l|l|}
\hline $\begin{array}{l}\text { Elément de } \\
\text { nature } \\
\text { (comparé=thème) A }\end{array}$ & \multicolumn{1}{|c|}{$\begin{array}{c}\text { Prédicat } \\
\text { de comparaison } \\
\text { B }\end{array}$} & $\begin{array}{l}\text { Elément de nature ou } \\
\text { outrel (comparant=phare) } \\
\text { C }\end{array}$ & $\begin{array}{c}\text { R. } \\
\text { d'impertinenc } \\
\text { e }\end{array}$ \\
\hline le soir & passant comme & la mort & $\mathrm{B} \neq \mathrm{A}$ \\
\hline le monde & s'appuie sur moi tel & un vieillard édenté & $\mathrm{B} \neq \mathrm{A}$ \\
\hline le soleil & passent comme & des obstacles & $\mathrm{B} \neq \mathrm{A}$ \\
\hline les nuages & chassele sable comme & une meute de chiens & $\mathrm{B} \neq \mathrm{C}$ \\
\hline le vent & change comme & les nuages & $\mathrm{B} \neq \mathrm{A}$ \\
\hline tout & retentit comme & un tambour & $\mathrm{A} \neq \mathrm{C}$ \\
\hline l'atmosphère & Bat comme & une métaphore du silence & $\mathrm{B} \neq \mathrm{A}$ \\
\hline l'atmosphère & & & $\mathrm{A} \neq \mathrm{C}$ \\
\hline
\end{tabular}

\section{IV.2.2 L'éthos de la poétesse en face de la nature}

Dans son deuxième remuement, Koltz adresse son éthos à la nature. Elle entame à se réfugier dans les éléments de la nature afin de comparer son tout au monde naturel et de respirer à travers la pleine fusion avec ses composants. Cette fusion se diversifie sous trois formes. Dans la première configuration de l'éthos, Koltz est inclue à un élément minéral: les pierres précieuses :

Mes pêchés

comptant comme

( ${ }^{1}$ ) Gabriel Urgel Reyes produit métaphore du silence album transdisciplinaire qui allie musique, dessins, poèmes et vidéo de sa création. 
La poétesse est-elle fière de ses péchés? Si les péchés sont une nature humaine. Koltz désire fort se débarrasser de cette nature pécheresse et s'allier à la nature, car il n'y a ni péché ni erreur dans celle-ci. À travers la structure comparative "pierres précieuses", elle recherche une composante de valeur pour l'être humain. Le recours à cette composante naturelle enchante visuellement le lecteur à travers les couleurs, les formes et la géométrie de ces pierres naturelles, ce qui s'applique au diamant, au saphir, au rubis et à l'émeraude. Par conséquent, la poétesse nous emmène dans un univers magique plein de beauté, à travers un choix concis et précis en utilisant cette comparaison avec le monde naturel.

Ensuite, la comparaison de l'éthos avec les matières solides de la terre se relie aux racines sous terre. Koltz dit :

\section{Mes paroles \\ travaillent la terre comme des racines souterraines $(P V: 28)$}

Mais pourquoi la poétesse a-t-elle choisi de comparer les mots de ses poèmes aux racines?

Premièrement, cette assimilation concrète de la botanique, reflète les dimensions environnementales à travers le verbe travailler au transitif direct, ce qui signifie que Koltz met en évidence le problème de la végétation verte afin de prendre soin donc au script Vert : une écriture poétique créative efficace envers l'environnement. Deuxièmement, la poétesse établit un siège de symbioses entre la valeur des racines de la plante et celle de l'écriture poétique de la vie humaine: sans racines, les plantes ne poussent pas et sans parole poétique de Koltz, il n'y a pas donc de créativité. Troisièmement, les lexèmes paroles et racines, au 
pluriel, convoquent l'étendue de la relation de bifurcation dans l'esprit des gens comme dans la terre.

La comparaison botanique crée donc une visualisation de l'approximation de l'image entre le comparé et le comparant, les deux origines de la vie : si les racines sont importantes pour la plante, la poésie est également importante pour l'humanité. Au final, l'épithète postposée souterraines se réfère au rôle des paroles comme des racines pour sauver l'humanité. Dans ce contexte, comparable à la botanique, nous pouvons supposer "le métabolisme poétique" dans le recueil qui signifie pour nous que les racines comme les paroles présentent des adaptations afin de faciliter le développement de la vie saine avec l'environnement. Pour Koltz, " la parole reste encore ce que l'humain possède de plus prometteur pour traverser le tunnel de la vie" (Frank, 2001, 893) pour s'intégrer à la nature.

Le long trajet à parcourir se complète lorsque Koltz s'est érigée en gardien de la nature dans une nouvelle comparaison complétant la précédente tirée de la botanique quand elle dit :

Comme un jardin

à l'abandon

je reste en éveil $(P V: 57)$

L'expression idiomatique être en réveil, remontée au Moyen-âge, qui signifie être sur ses gardes en état d'alerte, agite l'enthousiasme pour défendre la nature. Mais quel genre de nature? Le syntagme répandu chez les français un jardin à l'abandon s'attache "à définir les notions fondamentales nécessaires à la description des rapports entre sens et référence." (Melis, 1998) Koltz se rapporte à la nature sauvage en se référant à la composition jardin en friche afin d'élever son propre 
emblème vers la nature " : il faut laisser en friche les facultés de la nature. "

En harmonie complète avec la nature, la deuxième configuration de l'éthos fait référence à une autre partie du monde naturel ; celle animale dont la condition pathétique a été mise en double comparaison avec deux cas d'animaux extrêmement faibles. Koltz assimile son ombre à un poisson dans un filet de pêche ou à un oiseau d'une aile cassée en disant:

Je traîne mon ombre

comme une aile cassée (PV:20)

Je m'embourbe

dans mon ombre

comme un filet de pêche $(P V: 32)$

En effet, la reformulation poétique en rapport à une certaine contextualité, au fil du prolongement de la comparaison animale, donne sens au terme polysémique ombre dans la poésie de Koltz. Il revêt une forte occurrence stéréotypique interprétant l'écho naturel. Coincée avec son ombre, Koltz a tout le temps une obsession compulsive de : (son histoire, ses souffrances, son âge, l'âme de René, "son mari défunt" etc.)

Une pictographie écologique, cette ombre ralentit la cadence de la comparaison et la rend extrêmement lourde et agaçante à cause du choix des verbes traîne et m'embourbe. Ceuxci font référence à l'obstruction et l'incapacité de continuer la vie de la part d'une poétesse ayant 82 ans.

Il est clair que Koltz enchevêtre davantage le lecteur dans les réseaux écologiques dont elle-même est incapable de se libérer. La synecdoque aile cassée amplifie une paraphrase à la virtuosité de la poétesse en effectuant une interaction réciproque à 
travers une image consécutive négative. L'énonciatrice, impliquée volontairement par le pronom personnel je dans les griffes de la nature, accepte sa transformation en un oiseau aligné. Ce mécanisme sensuel conduit à d'autres transformations tout au long du recueil s'appuyant sur des formes d'intégration à la nature pour pouvoir y accéder. Koltz incite son lecteur à prendre part à ses moyens viables d'atteindre le contact intrinsèque dans le processus de représentation visuelle allégorique par une mémoire à accès naturel.

Épuisée de vagabondage dans l'espace naturel, Koltz termine, dans sa troisième configuration de l'éthos, les cercles de comparaison avec les tréfonds de la nature par deux cas de comparaison qui sont liés thématiquement à la mort de sorte que le sujet pluriel cendres "doit être lu avec la connotation de "restes" de manière définitive" (Row, 2012, 83). Ce terme connoté est donc devenu au centre de l'attention de la figure comparative sous ses différentes formes. Par conséquent, Koltz annonce :

\section{-Mes cendres descendront}

comme une pluie d'été

-Je passe à travers les siècles

comme un fragment

retournant vers les flammes

comme une étoile morte

$$
(P V: 110)
$$

La structure conflictuelle de PV fournit une poursuite de pluie d'été qui configure une référence précieuse dans la 
littérature française $\left({ }^{1}\right)$. Koltz a également reproduit ce syntagme nominal étrange sur les rivages de son dernier recueil en créant une écriture parallèle qui verse un état de vigilance intellectuelle soudaine comme la pluie d'été chez le lecteur.

Héroïquement, Koltz aborde la scène finale de sa vie dans laquelle le thème de /la mort/domine sa réflexion et ses idées. La poétesse a-t-elle peur de la mort? Elle avoue que " la mort ne [lui] fait pas peur, bien au contraire : ça [lui] fera un peu de repos !" (Entretien avec Cimatti, le 11/06/2018). L'écologie de comparaison se dépeint sous trois figures naturelles: pluies d'été, un fragment et étoile morte. Ceux-ci s'appuie sur le sème de /l'évanescence/ dont les entités sont bien éclairés : flammes et lumière s'accrochent à une coloration redondante d'une image interne et rituelle de la mort. L'oscillation entre l'espace géographique dans pluie \# la terre ou historique dans siècles n'a pas exempté Koltz de l'extrême concentration sur son chemin vers la mort.

De la sorte, les verbes descendront, passe, retournant, ne serait visible dépeignent son itinéraire fatal et doux sans hésitation. Ceux-ci constituent le dernier testament avant de périr. Les composants naturels se mêlent entre eux en tant que ingrédients d'une recette poétique reflétant la complexité de la dissolution de la poétesse à la nature, synthétisée ainsi d'après le modèle de Cohen (1968) :

(1) Pluie d'été, un roman de Marguerite Duras, reflète les valeurs littéraires sur l'enfance et l'immigration et porte aussi le nom du poème de Bonnefoy, dans son recueil Les Planches courbes en 2001, lié aux souvenirs d'enfance. 


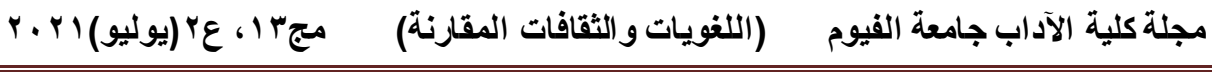

\begin{tabular}{|c|c|c|c|}
\hline $\begin{array}{l}\text { Elément de } \\
\text { nature } \\
\text { (comparé/ } \\
\text { thème) }=\mathbf{A}\end{array}$ & $\begin{array}{l}\text { Prédicat de } \\
\text { comparaison }=B\end{array}$ & $\begin{array}{l}\text { Elément de nature ou } \\
\text { outre }^{1} \text { (comparant/ } \\
\text { phare) }=\mathrm{C}\end{array}$ & d'impertinence \\
\hline mes pêchés & comptant comme & des pierres précieuses & $\mathrm{B} \neq \mathrm{C}$ \\
\hline je & $\begin{array}{l}\text { traîne mon ombre } \\
\text { comme }\end{array}$ & une aile cassée & $\mathrm{B} \neq \mathrm{A}$ \\
\hline mes paroles & $\begin{array}{l}\text { travaillent la terre } \\
\text { comme }\end{array}$ & des racines souterraines & $\mathrm{B} \neq \mathrm{A}$ \\
\hline je & $\begin{array}{l}\text { m'embourbe dans mon } \\
\text { ombre comme }\end{array}$ & un filet de pêche & $\mathrm{B} \neq \mathrm{A}$ \\
\hline mes cendres & descendront comme & une pluie d'été & $B \neq A$ \\
\hline je & reste en éveil comme & un jardin à l'abandon & $\mathrm{B} \neq \mathrm{C}$ \\
\hline je & passe comme & $\begin{array}{l}\text { un fragment retoumant } \\
\text { vers les flammes } \\
\text { une étoile morte dont la } \\
\text { lumière ne serait visible sur } \\
\text { la terre. }\end{array}$ & $\mathrm{B} \neq \mathrm{C}$ \\
\hline
\end{tabular}

\section{IV.2.3 Évocation de l'Autre : la nature, un médiateur spirituel}

La postmodernité de l'éthos de Koltz est un instrument d'agentivité dans son discours écopoétique. Un sujet parlant conditionné par les codes du langage poétique, elle engendre la dynamique avec l'Autre, René. Elle "projette un ethos qui lui permet d'entrer en relation avec l'autre." (Amossy, 2010, 63) Elle exerce ainsi intentionnellement sa volonté envers la nature traversée et pénétrée par la doxa écologique. Ce double statut du « je », à la fois agi et agissant, se manifeste dans son "le langage codé à l'extrême. " (Idem) Son ethos écopoétique produit ses effets identitaires en s'immergeant dans la parole poétique et poursuit son projet écopoétique précis.

René, ce nom propre "non-modifié et nu" (Jonasson, 1994, 134) dont le savoir métalinguistique et l'hypersémanticité 
crée "son mystérieux pouvoir de suggestion" (Pougeoise, 2005, 327) à travers un médium naturel, évoque alors inévitablement son souvenir dans la mémoire de la poétesse. Sa confiance illimitée dans la nature immortalise son René dans ses poèmes.

Bien que la culture occidentale n'approuve aucun désir contre l'individualisme, Koltz relève le défi en croyant en la multiplicité du soi. Cette croyance peut être attribuée à son mari: le stimulus de Koltz pour convoquer l'autre, le spiritisme de René. Le médium naturel se procure l'âme de l'autre pour adhérer à son esprit et à son corps. Mais qui est l'autre dans le recueil?

Certes, nous avons la conviction que l'Autre dans les poèmes de Koltz est René. Son grand amour [est] René, son mari." (Ehret, 2018) Ce prénom a pris trois synonymes dans PV: mon ombre, mon double et mon amant. Mon double remplace mon couple. La commutation de [d] au lieu de [K] excite une convergence phonémique entre double et deuil. Cette affinité acoustique de la continuité de la tristesse chez Koltz se ressent à cause de la perte de son mari. Elle lui dédie une séquence du recueil en disant :

\author{
À René \\ Ta solitude \\ ne m'appartient plus \\ je suis ton ombre \\ qui se déplace sans toi \\ tu es parti \\ sans partir \\ comment te rejoindre?(PV.74)
}

L'énoncé interrogatif comment te rejoindre? se connecte avec la nature sous trois formes sensorielles spécifiques: auditives, visuelles et tactiles, en invoquant des éléments excitants 
et spécifiques. Au départ, la poétesse se tourne vers le fleuve avec un stimulus audible en disant:

\section{Je t'écoute tel un fleuve \\ qui me traverse \\ et me suis $(P V: 67)$}

Les pronoms personnels esquissent finement la sphère de l'énonciation poétique : je me et te. Le verbe écouter approfondit l'acuité auditive grâce au comparant aquatique fleuve. Le fleuve, léger et silencieux, ravit à l'esprit de la poétesse les souvenirs longs avec son mari, René. Les sons du fleuve provoquent également une euphorie dangereuse de facteurs internes des verbes traverser et suivre Au moment où le bruissement léger du fleuve / René pénètre l'âme de la poétesse, tous les deux peuvent ainsi parcourir, s'avancer dans un état d'errance naturelle. Ce long voyage fait aboutir les deux amants à la mer où nous entendons d'autres sons naturels:

\section{Ta voix résonne encore en moi comme la mer dans un coquillage $(P V: 121)$}

L'image acoustique de l'analogie se complique jusqu'à ce que la poétesse se transforme à un coquillage au cœur de la mer / René. Ainsi, la voix de la mer est le catalyseur de l'image sonore dans ses oreilles comme son esprit. Le déferlement des vagues au coquillage exalte la puissance de la fréquence sonore de la mer: une forte imagination écopoétique.

Une autre similitude naturelle : la poétesse se transforme en un sol propice à la culture afin de donner à René des mots/ délicats, fruits /délicieux qui ressemblent à des plantes sauvages. Elle s'adresse à René en disant:

\section{À René}


Je te donnerai mes paroles végétant dans ma bouche

Comme des plantes sauvages (PV: 120)

La bouche met l'accent sur le sens du toucher, un sens extéroceptif qui excite une réaction tactile immédiate observable entre Koltz et René et se confond aussi avec le contact impliquant de mieux connecter la vision et l'audition entre eux. Le syntagme nominal plantes sauvages nous dirige vers une idée géniale dans $\mathrm{PV}$, à savoir que ses mots poussent sans intervention humaine. Il s'agit de poèmes postmodernes comme des plantes sauvages: tous les deux sont qualifiés d'indigènes. Leur cohérence "se fonde sur l'authenticité de la voix qui les porte [...] pour utiliser un terme de de la poétesse, sauvagerie." (Martínez, 2006, 139)

En plus, le contact direct avec la nature persiste le prolongement d'une expérience sensorielle les images comparatives. Le sème /goût/ est fort provoqué quand Koltz dit:

\section{Même la bouche de l'amant était froide comme un glaive (PV: 107)}

En fin de compte, toutes ses tentatives pour imposer l'Autre sont terminées; Koltz touche les lèvres de René, elle les trouve sans goût, sans sensation. La comparaison superpose le désespoir qui s'empare de Koltz, celui qui est évoqué par être froide, à un matériau rigide: glaive. Toutefois, la joie de la polysensorialité donne à cette totalité une comparaison étonnante. Koltz sollicite enfin René en disant:

\section{Laisse-moi goûter \\ Ton sang chaud}


Ta chair blanche

Brille dans l'obscurité

Comme un glacier. (PV:61)

La comparaison nous renseigne sur le positionnement de René. Les sens s'émeuvent autour d'un glacier, assimilant trop "vite ce lyrisme à un penchant vers le joli. [...] un goût de la sensation précise, à rebours des conventions." (Monte, 2018, 7)

Les verbes goûter et briller, les épithètes postposées chaud, blanche, et les lexèmes obscurité et glacier comblent le vide sensoriel de la perception de l'autre grâce à la polysensorialité : goût, toucher et vue. La poétesse convoque enfin le corps de René à travers le stimulus naturel sang chaud. L'acte directif : Laisse-moi goûter "implante l'autre en face de lui." (Benveniste, 1974, 82) PV se donne " sous la forme du sensible [...] dans l'esprit [...] séjourné dans les sens »(Le Breton, 2006, cité par Cottereau, 2012, 19) ravissants et contradictoires envers René regroupés dans le tableau ci-dessous toujours selon le modèle de Cohen :

\begin{tabular}{|l|l|l|c|}
\hline $\begin{array}{l}\text { Elément de } \\
\text { nature(comparé } \\
\text { =thème) A }\end{array}$ & $\begin{array}{l}\text { Prédicat de } \\
\text { comparaison } \\
\text { B }\end{array}$ & $\begin{array}{l}\text { Elément de nature ou outre } \\
\text { (comparant=phare) } \\
\text { C }\end{array}$ & $\begin{array}{l}\text { R. } \\
\text { d'impertinence }\end{array}$ \\
\hline Je & t'écoute tel & $\begin{array}{l}\text { un fleuve qui me traverse et } \\
\text { me suis }\end{array}$ & $\mathrm{B} \neq \mathrm{C}$ \\
\hline $\begin{array}{l}\text { la bouche de } \\
\text { l'amant }\end{array}$ & était froide comme & un glaive & $\mathrm{B} \neq \mathrm{A}$ \\
\hline $\mathrm{Je}$ & $\begin{array}{l}\text { te donnerai mes } \\
\text { paroles végétant } \\
\text { dans ma bouche } \\
\text { comme }\end{array}$ & Comme des plantes sauvages & $\mathrm{B} \neq \mathrm{A}$ \\
\hline Ta voix & $\begin{array}{l}\text { résonne encore en } \\
\text { moi comme }\end{array}$ & la mer dans un coquillage & $\mathrm{B} \neq \mathrm{A}$ \\
\hline
\end{tabular}

$P V$ laisse les traces à la charge connotative dont les comparants empruntés au répertoire du projet naturel se réinventent par l'intuition d'une poétesse écologiste. 


\section{Conclusion}

Tout en donnant un aperçu de la dissolution dans la nature, $P V$ est une manifestation de la philosophie de Koltz pour atteindre cette conscience. Koltz avait son habilité linguistique de communication appropriés, susceptibles d'embrasser et d'être embrassée par la nature. Ceci est pour instituer une connexion physique avec le monde naturel puisque le terme dissolution est le synonyme chez Koltz de recréer, et non pas détruire, l'élément naturel ainsi que le soi.

Chaque page dans le recueil est un coup de feu de la dissolution sans faille de la poétesse dans le monde naturel qui s'unit en un tissu unique. Nombreux corps naturels imposent à la poétesse un changement constant de l'espace et du décor, un défi mettant en avant le pouvoir descriptif de Koltz et sa maîtrise de la technique écopoétique.

$P V$, à travers cette étude, se transforme en un glossaire environnemental rempli de la vitalité du lexique qui forme la conscience de la poétesse et son immersion dans une joie totale d'intégration avec les éléments de l'environnement: verbes géographiques tels que traverser, s'envoler, s'intégrer, creuser la distance, trainer, se réfugier, végéter, etc., les verbes géohistoriques tels que descendre de, vivre, devancer, charrier, etc., l'espaces naturels Nil, Alexandrie, Babel, soleil, étoile(s), ciel, enfer, océan, vacarme, tempête, etc., les animaux migratoires, troupeau, Anubis, plume, mouettes, lézard, etc., les formes de géométrie naturelle triangle, hamac, flocons, structures, falaises, formes, caravanes. Tous ces éléments se sont reposés sur quatre moyens naturels de déplacement: ailes, nageoires, tentacules, antennes.

$P V$ marque également l'apogée de la nature depuis le retrait du niveau géographique pour laisser place à l'émotionnel. Voici un train d'images illustrant le processus d'immersion dans la 
nature. La combinaison de la métaphore et celle de la comparaison montre à quel point la nature et la poétesse ont soif de s'embrasser. Ces images sont l'incarnation de la philosophie de la vie de Koltz. Les deux images (métaphores et comparaison) avec d'autres images secondaires telles que métonymie, synecdoque et analogie, oxymore, reflètent, en outre, le sens de l'humilité qui intensifie toujours le désir des poètes écologiques de se fondre dans le monde des non-humains. La joie que Koltz trouve dans la nature peut amener le lecteur à penser qu'elle a la même attitude que cette nature.

Pour Koltz le corps est la première entrée dans le mental. En outre, pour devenir un Autre, il faut un contact sensuel avec l'élément ciblé du corps censé avoir la présence d'un tel élément plutôt que de l'esprit. Pour transmettre sa pensée, la poétesse frappe d'abord le sens auditif du lecteur. Ensuite, elle suscite le sens du toucher. Elle incite finalement le sens de la vue. Étant donné que les pouvoirs d'observation de Koltz deviennent son accès à se sentir dissolue totalement au monde naturel, il s'agit donc d'un lien surnaturel / métaphysique qui s'ensuit dans le jeu savant des sens: la vue, de l'ouïe, du toucher, et du goût.

L'érotisation du langage (Monte, 2018) poétique de Koltz dans $P V$ s'est opérée par les chaînes des allitérations dans $[V],[t],[n],[s],[l],[k],[p],[b],[d]$ et assonances dans $[a],[e]$, $[\varepsilon],[\partial],[o][\tilde{O}]$. Cette érotisation densifie l'écholalie naturelle par sa récurrence presque cacophonique. Ces récurrences phoniques du recueil se sont réunies sous le signe associé à une organisation écopoétique interne des vers irréguliers pour reproduire un effet perceptible des chaînes phoniques exemplifiant l'unité du recueil-album. L'apparente harmonie coulée trouve sa raison de la priorité donnée à l'architecture sonore et à ses effets d'écho naturel. 
Koltz a présenté un modèle unique de l'écopoésie qui a montré une grande différence entre la poésie romantique et celle environnementale américaine. Dans le cas de la poésie romantique, les poètes, tels que Victor Hugo et Charles Baudelaire, conservent leur humanité face à la nature, qui, dans ce cas-là, est un espace de repos et de contemplation. Dans le cas de la poésie environnementale américaine, nous veillons à ce que les poètes, tels que Henry David Thoreau et Mary Austin, ne se soucient que de se mêler à l'espace américain. Contrairement aux deux cas précédents, Koltz a spontanément libéré l'imagination de sa poésie environnementale afin de se déplacer avec un confort total dans des horizons divers et variés en adoptant une déconstruction de l'espace naturel. 


\section{Références bibliographiques}

\section{Corpus de l'étude}

Anise Koltz, A. (2018), Pressée de vivre, Arfuyen, OrbeyParis.

\section{II. ouvrages de Koltz cités dans l'article}

Anise Koltz, A. (2009) Lune noircie, Arfuyen, Orbey-Paris. (2012) Soleils chauves, Arfuyen, Orbey-

Paris.

(2013) Galaxies intérieures, Arfuyen, Orbey-

Paris.

(2015) un monde de pierres, Arfuyen, Orbey-

Paris.

\section{Articles et périodiques en ligne- entretiens avec Anise Koltz:}

- André Guilcher, A. (1954), Morphologie littorale et sousmarine, PUF, Paris.

- Anise Koltz, La poétesse luxembourgeoise lauréate du Goncourt de la poésie in La dépêche du midi. Fr. vendredi 17 avril 2020, Saint Etienne, Relax News

- Blanc, N. (2008/2), Chartier, D. et Pughe, Th., Littérature $\&$ écologie : vers une écopoétique in Écologie \& politique $\left(\mathrm{N}^{\circ} 36\right)$.

- Bouvet, D. (1997), Le corps et la métaphore dans les langues gestuelles : à la recherche du mode de production des signes, L'Harmattan, in (coll. « Sémantiques »).

- Buekens, S. (2019), " L'écopoétique : une nouvelle approche de la littérature française », Elfe XX-XXI [En 
ligne], 8 | 2019, mis en ligne le 10 septembre, consulté le 13 septembre 2019.

- Cohen, J. La comparaison poétique : essai de systématique. In: Langages, $3^{\mathrm{e}}$ année, $\mathrm{n}^{\circ} 12,1968$. Linguistique et littérature.

- Constantin de Chanay, H. et Rémi-Giraud, S. (2002) « « Espèces d'espaces »: approche linguistique et sémiotique de la métaphore », Mots. Les langages du politique [En ligne], 68 |, mis en ligne le 30 avril 2008.

- Cottereau, A. (2012), « Habiter par corps. Éducation relative à l'environnement, in Regards-RecherchesRéflexions », vol. 10, p. 17-34.

- Deschamps, C. (2015), «Pierre Schoentjes, Ce qui a lieu. Essai d'écopoétique, Marseille, Wildproject, coll. "Tête nue », 2015 », Essais [En ligne], 13 | 2018, mis en ligne le 01 décembre 2019, consulté le décembre 2019. URL : http://journals.openedition.org/essais/492

- Ehret, J. (2018). "Dieu" dans la poésie d'Anise Koltz, 6e leçon du cours "Spiritualités en dialogue", 21 juin 2018, www.Isrs.lu.

- Finck, M. et Ergal, Y., M. (2019), Anise Koltz, L'inapaisée, La poésie entre les langues, Collection : Espaces Littéraires: Littérature, Documents, Récits.

- Frank, W. (2001), L'écrivain francophone grand-ducal et ses choix linguistique et culturel. In: Revue Belge de philologie et d'histoire, tome 79 , fasc. 3, Langues et littératures modernes.

- Giuliani, P. (2008), Le sang classique entre histoire et littérature : hypothèses et propositions, in Dix-septième siècle 2008/2 (n² 239). 
- Gomez, B. A, (2006), L'apport de la linguistique dans l'approche au sens du poème, Linguistique plurielle, in Valencia. 25, 26 et 27 Octobre.

- Jean-Pierre Jossua, J.,P., (2014/1) Bulletin de théologie littéraire, in Revue des sciences philosophiques et théologiques 2014/1 (Tome 98).

- M. Carel et O. Ducrot, (1999) Le problème du paradoxe dans une sémantique argumentative, in Langue française No 123, Année

- Mel'čuk, I., et Polguère, A. (2009), « Prédicats et quasiprédicats sémantiques dans une perspective lexicographique », Lidil [En ligne], 37 | 2008, mis en ligne le 01 septembre 2009, consulté le 27 mars 2020. URL : http://journals.openedition.org/lidil/2691 ; DOI : https://doi.org/10.4000/lidil.2691.

- Melis, L. (1998), Réflexions sur la structure syntaxique du syntagme nominal, in La ligne claire.

- Michèle, M. (2007), L'oxymore : figure syntacticosémantique ou élément d'une stratégie para-doxique? Université de Toulon, Babel EA 2649, ILF-CNRS UMR 6039 L'Analyse pragma-énonciative des figures. Journée CONSCILA du 19 octobre.

- ------------------, «L'éthos en poésie contemporaine : le cas de James Sacré et d'Antoine Émaz».Babel. In Littératures plurielles 34.

- -------------, (2018). Pour une approche linguistique de la poésie contemporaine : à la rencontre de James Sacré et d'Ariane Dreyfus, in Pratiques.

- Miñano Martínez, E. (2006), Le silence dans la poésie d'Anise Koltz: de chants au refus à béni soit le serpent, Université de València in Quaderns de Filologia. Etudes littéraires. Vol. XI (2006). 
- Nathalie, B., Denis, CH. Pughe, Th. (2008/2), Littérature \& écologie : vers une écopoétique », Ecologie \& politique, $\mathrm{n}^{\circ}$ 36, p. 15-28. DOI : 10.3917/ecopo.036.0015 : https: //www.cairn.info/revue-ecologie-et-politique1.

- Ntsame Okourou, F. (2018), Pour une écocritique du discours littéraire gabonais, http://www.regalish.net / Numéro : 4, décembre 2018 / ISSN 2520-9809.

- Passeron, J. Cl. (2000), Analogie, connaissance et poésie Revue européenne des sciences sociales T. 38, No. 117, Métaphores et Analogies schèmes argumentatifs des sciences sociales: Ve Séminaire interdisciplinaire du Groupe d'Etudes "Raison et rationalités."

- Pelechová, J. (2013/3), La scénographie, in Études théâtrales $\left(\mathrm{N}^{\circ}\right.$ 58), Mis en ligne sur Cairn.info le 01/01/2018.

- Poivret, PH. (2020), Anise Koltz : une poésie de la vie sans concession, in Passaparola, Pro/p(r)ose Magazine | Numéro exceptionnel et thématique.

- Pughe, Th. (2005), Réinventer la nature : vers une écopoétique, ÉA 58-1in: 68-81. Klincksieck, in 'Études anglaises", Didier Érudition.

- Riffaterre, M. (1971), Sémantique du poème, Cahiers de l'AIEF Michel Barrucand, L'espace en poésie - poésie de l'espace https://doi.org/10.4000/caliban.

- Van de Velde, D. (2009/3), Les adverbes de manière : propriétés inhérentes et propriétés héritées des prédicats verbaux, in Langages $\left(n^{\circ} 175\right)$

\section{Ouvrages consacrés à la linguistique et écolinguistique}

- Adam, J. M. et Petitjean, A. (1989). Le texte descriptif, Nathan, Paris. 
- Adam, J.M., (1992), Les textes: types et prototypes, A. Colin, Paris.

- Benveniste, E. (1974), Problèmes de linguistique générale, tome II, Gallimard, Paris.

- Buell, L, (1995), The Environmental Imagination, Harvard UP, London.

- Buell, L. et Suberch icot, A. (2002), Littérature américaine et écologie, Le Harmattan, Paris.

- Calvet, L.-J. Pour une écologie des langues du monde, Plon, Paris, 1999.

- Dessons, G. (2005), Introduction à la poétique: approche des théories de la littérature, Armand Colin, Paris.

- Fischesser, B. et M.-F. Dupuis-Tate. 1996. Le Guide illustré de l'écologie. : Éditions de la Martinière, Paris.

- Fontanier, P. (1977), Les figures du discours, Champs Flammarion, Paris.

- Forest, Ph. (1989), le symbolisme ou naissance de la poésie moderne, Pierre Bordas et fils, Paris.

- Jakobson, R. (1970), Essais de linguistique générale, Minuit, Paris.

- Jakobson, R. (1973), Questions de poétique, Seuil, Paris

- Jonasson, K. (1994). Le nom propre: Construction et interprétation, Duculot, Paris.

- Kristeva, J., (1974), La Révolution du langage poétique, Seuil, Paris,

- Le Breton, D. (2006), La Saveur du monde. Une anthropologie des sens, 2006, cité par A. Cottereau, 2012.

- Le Breton, D. (2006), La Saveur du monde. Une anthropologie des sens, Métailié, Paris.

- Lechevrel, N. (2008), L'approche écologique en linguistique : le cas de l'écolinguistique, Thèse de doctorat en Sciences du langage - Linguistique, à Paris, EHESS. 
- Molino, J. et Gardes-Tamine, J. (1982), Introduction à l'analyse de la poésie, vers et figures, PUF, Paris.

- Mounin, G., (1971) la communication poétique, Gallimard, Paris.

- Poupon, F. (2019), « Géopoétique et écologie dans l'œuvre poétique de Kenneth White », Essais 13 | 2018, mis en ligne le 01 décembre 2019, consulté le 12 décembre 2019.

- Row, J. (2012), Aux cendres d'un époux " traumatisme et temps du désir inexprimable dans Andromaque de Racine, édité par Alastier Hemmens et Russell Williams.

- Ruth, A. (2010), La présentation de soi L'Interrogation philosophique, P UF, Paris.

- Ruth, A. (2016), L'argumentation dans le discours, pour la nouvelle présentation, Armand Colin, Paris.

- Zucca, M. (2015), La migration des oiseaux : Comprendre les voyageurs du ciel, éditions Sud-Ouest.

\section{Dictionnaires}

- Dictionnaire des auteurs luxembourgeois, en ligne avec la coopération de l'entreprise magic moving pixel.

- Dubois, J., Marcellesi,J. B. et Mével, J. P. (1994). Dictionnaire de linguistique et des sciences du langage (Trésors du français), Larousse, Paris.

- Pougeoise, M. (2005). Dictionnaire de poétique, Belin, Paris,

- Robert, P. et Rey-Derove, J. (2012), Le Petit Robert, Dictionnaire alphabétique et analogique de la langue française, nouvelle édition, Dictionnaires, Le Robert, Paris. 


\section{الذوبان في الطبيعة: دراسة لغوية بيئية لايوان

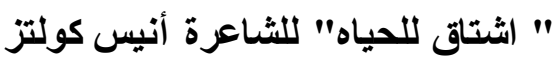 \\ ملخص}

يهذف هذا العمل إلى دراسة لغة النظم الثعري عند أنيس كولنز بوصفها

شاعرة استطاعت أن تمتزج كليةً بل وتذوب في عالم الطبيعة. وذلك من خلال تحليل خصائص وسمات لغة شعرالبيئة المستخدمة بشكل جلي في ديوانها الأخير "مشتاقة للعيش"2018. ويسلط هذا العمل الثعري الضوء على تعلق الثاعرة الثديد بالطبيعة وكوامنها وعلى نهجها في التعبير عن الثعور بالارتباط الوثيق بالطبيعية وذلك من خلال استخدام مفردات لغوية تتعلق بالبيئة ذاتها ومكوناتها المختلفة. وركزت الدراسة على قدرة الثاعرة الفائقة في استخدام مختلف الحواس للوصول إلى عمق عالم الطبيعة، وكذلك مهارتها في الاستعانة ببعض الأساليب اللغوية الفربدة، والتي قد ساعدنها على الارتقاء بفنها الشعري وجذب انتباه القارئ. كما أبرزت الدراسة دقة الوصف وانقان إنشاء التراكيب اللغوية المناسبة لدى الثناعرة لاسيما وقت التتقل بين عناصر الطبيعة المختلفة. خلاصة القول، تحاول الدراسة إظهار موقف أنيس كولتز من الطبيعة وأثز ذلك في فن نظمها الشعري، بالإضافة إلى محاولة الوقوف على ماتم استخدامه من أدوات وأساليب جديدة من أجل صياغة صورة شعرية مختلفة لعالم الطبيعة، مما يعد نهجاً لغوياً جديداً يسمى تحديداً علم اللغة البيئي (الإيكولوجي) 